\title{
Dendritic Morphology and Inhibitory Regulation Distinguish Dentate Semilunar Granule Cells from Granule Cells through Distinct Stages of Postnatal Development
}

\author{
Akshay Gupta ${ }^{1,3}$, Archana Proddutur ${ }^{1,3}$, Yun-Juan Chang ${ }^{2}$, Vidhatri Raturi ${ }^{1}$, Jenieve \\ Guevarra $^{1}$, Yash Shah ${ }^{1}$, Fatima S. Elgammal ${ }^{1}$, and Vijayalakshmi Santhakumar ${ }^{1,3}$ \\ ${ }^{1}$ Department of Pharmacology, Physiology and Neuroscience, ${ }^{2}$ Office of Advance Research \\ Computing, Rutgers New Jersey Medical School, Newark, New Jersey 07103, ${ }^{3}$ Department \\ of Molecular, Cell and Systems Biology, University of California Riverside, California \\ 92521.
}

ORCHID ID: Gupta A (0000-0002-3788-6277); Proddutur A (0000-0003-2333-4188); Chang Y-J (0000-0003-2688-6391); Santhakumar V (0000-0001-6278-4187)

Short Title SGCs during postnatal development

Word count for Abstract: $243 \quad$ Number of pages: $42 \quad$ Number of figures: 6

\section{Declarations:}

Funding: The project was supported by National Institutes of Health/National Institute of Neurological Diseases and Stroke R01 NS069861, R01NS097750 and New Jersey Commission on Brain Injury Research CBIR16IRG017 to V.S. and, CBIR11FEL003 to A.G

Conflicts of interest/Competing interests: No conflicts or competing interests to discuss

Availability of data and material: All reconstructions will be uploaded to Neuromorpho.org. Physiology data will be shared upon request.

Code availability: Standard software packages, Neurolucida 360, pClamp10 and R were used in analysis. Custom IGOR-Pro code for IPSC analysis will be made available upon request.

Authors' contributions: AG, JG, and AP performed experiments; AG, Y-JC, YS, and VR analyzed data; AG, DS, FSE, and VS interpreted results of experiments; AG, and AP prepared figures; AG and VS conceived of and designed research; AG, and VS drafted manuscript.

Acknowledgements: We thank Dr. Luke Fritzky at the Rutgers Imaging Core for help with imaging and Dipika Sekhar for data entry. We thank Drs. Deepak Subramanian and Kelly A. Hamilton for thoughtful discussions and comments.

Correspondence: $\quad$ Vijayalakshmi Santhakumar, $\mathrm{PhD}$

Department of Molecular, Cell and Systems Biology

University of California, Riverside

E-mail: vijayas@ucr.edu 


\section{Abstract}

3 Semilunar granule cells (SGCs) have been proposed as a morpho-functionally distinct class of

4 hippocampal dentate projection neurons contributing to feedback inhibition and memory

5 processing in juvenile rats. However, the structural and physiological features that can reliably

6 classify granule cells (GCs) from SGCs through postnatal development remain unresolved.

7 Focusing on postnatal days 11-13, 28-42, and >120, corresponding with human infancy,

8 adolescence, and adulthood, we examined the somatodendritic morphology and inhibitory

9 regulation in SGCs and GCs to determine the cell-type specific features. Unsupervised cluster

10 analysis confirmed that morphological features reliably distinguish SGCs from GCs irrespective

11 of animal age. SGCs maintain higher spontaneous inhibitory postsynaptic current (sIPSC)

12 frequency than GCs from infancy through adulthood. Although sIPSC frequency in SGCs was

13 particularly enhanced during adolescence, sIPSC amplitude and cumulative charge transfer

14 declined from infancy to adulthood and were not different between GCs and SGCs.

15 Extrasynaptic GABA current amplitude peaked in adolescence in both cell types and was

16 significantly greater in SGCs than in GCs only during adolescence. Although GC input

17 resistance was higher than in SGCs during infancy and adolescence, input resistance decreased

18 with developmental age in GCs while it progressively increased in SGCs. Consequently, GCs

19 input resistance was significantly lower than SGCs in adults. The data delineate the structural

20 features that can reliably distinguish GCs from SGCs through development. The results reveal

21 developmental differences in passive membrane properties and steady state inhibition between

22 GCs and SGCs which could confound their use in classifying the cell types.

23 Keywords: inhibition, GABA, dentate gyrus, extrasynaptic, development, granule cell,

24 semilunar granule cell 
25 The dentate gyrus, the primary gateway for cortical inputs to the hippocampus, plays a unique

26 role in memory processing as a center for sparse coding, mediated by the hyperpolarized resting

27 membrane potential of granule cells as well as the robust synaptic and extrasynaptic GABAergic

28 inhibition (Dengler and Coulter 2016). The classical dentate projection neurons, granule cells

29 (GCs), which number in over a million in the rat brain, rest at more hyperpolarized potentials

30 than most hippocampal neurons, receive powerful feedback inhibition, and project strong

31 "detonator" synapses to CA3 enabling sparse yet reliable transmission (Dengler and Coulter

32 2016; Engel and Jonas 2005). Recent characterization of semilunar granule cells (SGCs), a

33 dentate cell-type originally identified by Ramón y Cajal (1953), has revealed a second class of

34 dentate projection neurons that are located in the inner molecular layer (IML), has distinctive

35 somato-dendritic structure, persistent firing, and robust inhibition (Gupta et al. 2012; Larimer

36 and Strowbridge 2010; Williams et al. 2007). While SGCs have been physiologically defined in

37 rat (Gupta et al. 2012; Williams et al. 2007), cells with dendritic morphology consistent with

38 SGCs have been observed in primates (Duffy and Rakic 1983; Seress and Frotscher 1990).

39 Additionally, physiological recordings from cells with morphological features of SGCs have

40 been performed in mouse and rabbit (Sancho-Bielsa et al. 2012; Save et al. 2018), indicating

41 their presence across mammalian species. Due to their unique persistent firing in response to

42 inputs, SGCs have been proposed to play a crucial role in regulating GC feedback inhibition,

43 sparse coding of inputs, and pattern separation (Larimer and Strowbridge 2010; Walker et al.

44 2010). Recent studies suggest that SGCs are preferentially involved in memory engrams (Erwin

45 et al. 2020). Since SGCs lack a cell-specific neurochemical marker, morphological

46 characteristics, and lower input resistance $\left(R_{\text {in }}\right)$ are currently the primary approach to identify 
47 SGCs. However, the limited literature on SGC morphology and synaptic inputs during

48 development have impeded further analysis of the role of SGCs in the dentate circuit.

49 Developmentally, both SGCs and GCs express the homeodomain transcription factor Prox1

50 (Gupta et al. 2012) and derive from the same neural precursor pool, although SGCs differentiate

51 in a more restricted embryonic phase, demonstrating a common lineage between SGCs and GCs

52 (Kerloch et al. 2018; Save et al. 2018). Based on the embryonic developmental analysis, SGCs

53 are estimated at $3 \%$ of the neurons generated from the dentate neurogenic pool from which GCs

54 derive (Save et al. 2018). Like GCs, SGCs have dendrites in the dentate molecular layer, and

55 axons projecting to CA3. However, they can be distinguished from GCs based on their expansive

56 dendritic span, large semi-lunar somata in the inner molecular layer (IML), and frequent

57 presence of IML axon collaterals (Gupta et al. 2012; Williams et al. 2007). In earlier studies in

58 rats, we defined SGCs on the basis of their wider dendritic angle (Gupta et al. 2012), which was

59 subsequently confirmed in mice (Save et al. 2018). To date, morphometric analysis of SGCs has

60 been limited to a narrow window of postnatal day (PD) 14-42 in rats, which is consistent with the

61 neurological developmental state of human adolescence (Semple et al. 2013; Sengupta 2013), or

62 in an embryonically labeled subgroup of neurons in young adult mice (Save et al. 2018).

63 However, whether SGCs retain their distinct structural characteristics through postnatal

64 development has not been examined. Recent studies have suggested layer specific differences in

65 GC morphology (Kerloch et al. 2018) and disease-related changes in GC dendritic features

66 (Freiman et al. 2011; Llorens-Martin et al. 2015; von Campe et al. 1997). However, without

67 knowing the specific structural features that distinguish GCs from SGCs, it is difficult to

68 interpret whether reports on changes in GC morphology reflect inclusion of structurally distinct

69 SGCs in the datasets. Moreover, the ability to reliably distinguish SGCs from GCs across animal 
70 age is a prerequisite to elucidating the unique role that SGCs play in dentate processing.

71 Unsupervised analysis of dendritic morphometric features is ideally suited to examine whether

72 SGCs remain distinct from GCs through development and to elucidate the somato-dendritic

73 features that specify the cell types.

74 Physiologically, SGCs show prolonged firing, reduced spike frequency adaptation, and lower $\mathrm{R}_{\text {in }}$

75 than GCs (Gupta et al. 2012; Save et al. 2018; Williams et al. 2007). Unlike GCs, SGCs respond

76 to perforant-path stimulation with persistent firing lasting several seconds, which correlates with

77 periods of increased hilar activity termed "up-states" (Larimer and Strowbridge 2010). SGCs

78 have larger NMDA currents (Williams et al. 2007) than GCs, make synaptic contacts with hilar

79 mossy cells and interneurons and have been proposed to drive granule cell feedback inhibition

80 (Larimer and Strowbridge 2010). Apart from excitation, we previously demonstrated that SGCs

81 have greater synaptic and extrasynaptic GABA currents (Gupta et al. 2012). Additionally, we

82 identified that GCs and SGCs have diametrically opposite changes in synaptic and tonic GABA

83 currents after brain injury suggesting that differences in inhibition which could contribute to

84 distinct roles for SGCs and GCs in the dentate circuit. GCs undergo changes in both tonic and

85 synaptic GABA currents during postnatal development (Hollrigel et al. 1998; Holter et al. 2010;

86 Lee and Liou 2013). In parallel, studies on adult-born GCs labeled at specific time points show

87 cell-age dependent maturation of GABA currents, ultimately reaching the levels similar to

88 mature GCs in age-matched animals (Dieni et al. 2012; Li et al. 2012). Yet, whether inhibition

89 in SGCs remains distinct from mature GCs through postnatal development is not known. While

90 GCs show prolonged functional maturation ( $\mathrm{Yu}$ et al. 2013a), age-dependent changes in

91 inhibitory regulation of SGCs remains to be tested. This study was conducted to explicitly to

92 determine the characteristic structural features which can be used to distinguish SGCs from GCs 
93 through postnatal developmental stages representing infancy ( PD 11-13, prior to rodent eye

94 opening), adolescence (PD 28-42 days, period of cortical maturation), and adult (> PD 120,

95 cortical and sexual maturity) stages of development (Semple et al. 2013) and to identify the

96 differences in steady-state inhibitory regulation of the two cell types through maturation of the

97 dentate circuit. 


\section{Materials and Methods}

99

100

101

102

103

104

105

106

107

108

109

110

111

112

113

114

115

116

117

Animals. All experiments were performed in accordance with IACUC protocols approved by Rutgers-NJMS, Newark, NJ, and the University of California at Riverside, CA and in keeping with the ARRIVE guidelines. The study included Wistar rats ranging in age from 11-13 days, 28-42 days, and 120-180 days designated as infancy, adolescence, and adulthood, respectively (Semple et al. 2013; Sengupta 2013). Due to the potential effects of hormonal variation on GABA currents, slice recordings in rats from $>28$ days were restricted to males. A subset of recordings was derived from surgical or saline-injected controls for an independent study and was pooled with data from naïve rats from which they showed no statistical difference.

Slice Physiology. Rats were anesthetized with isoflurane and decapitated. Horizontal brain slices $(300 \mu \mathrm{m})$ were prepared in ice-cold sucrose artificial cerebrospinal fluid (sucrose-aCSF) containing the following (in $\mathrm{mM}$ ): $85 \mathrm{NaCl}, 75$ sucrose, $24 \mathrm{NaHCO}_{3}, 25$ glucose, $4 \mathrm{MgCl}_{2}, 2.5$ $\mathrm{KCl}, 1.25 \mathrm{NaH}_{2} \mathrm{PO}_{4}$, and $0.5 \mathrm{CaCl}_{2}$ using a Leica VT1200S Vibratome. The low-sodium sucroseaCSF has been shown to improve interneuron viability in adult slices (Tanaka et al. 2008). The slices were sagittally bisected and incubated at $32^{\circ} \mathrm{C}$ for $30 \mathrm{~min}$ in a submerged holding chamber containing an equal volume of sucrose-aCSF and recording aCSF, and subsequently were held at room temperature $\left(22-23^{\circ} \mathrm{C}\right)$ prior to recordings. The recording aCSF contained the following (in $\mathrm{mM}): 126 \mathrm{NaCl}, 2.5 \mathrm{KCl}, 2 \mathrm{CaCl}_{2}, 2 \mathrm{MgCl}_{2}, 1.25 \mathrm{NaH}_{2} \mathrm{PO}_{4}, 26 \mathrm{NaHCO}_{3}$, and 10 D-glucose. All solutions were saturated with $95 \% \mathrm{O}_{2}$ and $5 \% \mathrm{CO}_{2}$ and maintained at a $\mathrm{pH}$ of 7.4 for $1-6 \mathrm{~h}$. Slices were transferred to a submerged recording chamber and perfused with oxygenated aCSF at $33^{\circ} \mathrm{C}$. Whole-cell voltage-clamp recordings from putative GCs in the dentate granule cell layer (GCL) and SGCs in the inner molecular layer (IML) were performed using infrared differential interference contrast visualization techniques (Gupta et al. 2012; Yu et al. 2016) with a Nikon 
121 Eclipse FN-1 microscope, using a 40X, 0.80 NA water-immersion objective. Recordings were

122 obtained at least $50 \mu \mathrm{m}$ below the slice surface in a field with several viable hilar neurons,

123 typically within 1-6 hours after dissection in infant/adolescent mice and 1-3 hours following

124 dissection in adult mice to ensure optimal slice health. Analysis was restricted to cells with

125 resting membrane lower than $-60 \mathrm{mV}$. Recordings were obtained using MultiClamp 700B

126 (Molecular Devices). Data were low pass filtered at $3 \mathrm{kHz}$, digitized using Digidata 1440A, and

127 acquired using pClamp10 at $10 \mathrm{kHz}$ sampling frequency. Tonic and synaptic GABA currents

128 were recorded in aCSF with no added GABA or GABA transporter antagonists in the recording

129 solution (Gupta et al. 2012; Yu et al. 2013b). Voltage-clamp recordings of inward GABA

130 currents were obtained from a holding potential of $-70 \mathrm{mV}$ using microelectrodes (5-7 $\mathrm{M} \Omega$ )

131 containing (in $\mathrm{mM}$ ): $125 \mathrm{CsCl}_{2}, 5 \mathrm{NaCl}, 10 \mathrm{HEPES}, 2 \mathrm{MgCl}_{2}, 0.1$ EGTA, $2 \mathrm{Na}-\mathrm{ATP}$, and 0.5

132 Na-GTP, titrated to a pH 7.25 with $\mathrm{CsOH}$. Biocytin (0.2\%) was included in the internal solution

133 for post hoc cell identification, and the glutamate receptor antagonist kynurenic acid (3 mM

134 KynA, Tocris Bioscience) was included in the external solution to isolate GABA currents.

135 Passive membrane properties including resting membrane potential and $\mathrm{R}_{\text {in }}$ were recorded in

136 current clamp using an internal containing (in mM) $126 \mathrm{~K}$-gluconate, $4 \mathrm{KCl}, 10 \mathrm{HEPES}, 4 \mathrm{Mg}$ -

137 ATP, 0.3 Na-GTP, 10 Phosphocreatine and 0.2\% biocytin (Gupta et al. 2012). Among neurons

138 recorded in the IML, only cells showing obtuse dendritic angles, presence of dendritic spines and

139 axon projecting to the hilus (Gupta et al. 2012) (Supplementary Figure 1) were included in the

140 physiological analysis as putative SGCs. The somata of all GCs included in the analysis were

141 located in the GCL and spanned the entire extent of the GCL (Supplementary Figure 2). Neurons

142 with small somata located in the subgranular zone, putative immature granule cells, were not

143 included in the analysis. Recordings were discontinued if series resistance increased by $>20 \%$. 
144 Access resistance was not different between cell-types or between developmental groups and

145 analysis of access resistance failed to reveal systematic differences recordings in between

146 adolescent and adult slices (Access resistance in M $\Omega$ in GC and SGC combined, Adolescent

$14721.6 \pm 1.8$ and Adult $20.9 \pm 1.7, \mathrm{p}=0.8$ by $t$ test) indicating consistency in slice health. Following

148 establishment of whole cell mode, baseline recordings were obtained for a minimum of five

149 minutes in the presence of KynA prior to addition of GABA blockers. Recordings with excessive

150 baseline fluctuations during the 3-5 minutes of recordings in KynA were discarded. All salts

151 were purchased from Sigma-Aldrich (St. Louis, MO). Tonic GABA current, the steady-state

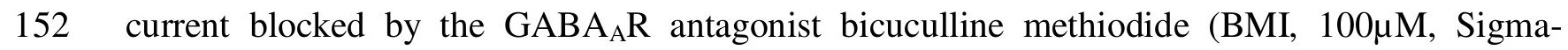

153 Aldrich) or gabazine (SR95531, $10 \mu \mathrm{M}$, Sigma-Aldrich), was measured as described previously

154 (Gupta et al. 2012; Yu et al. 2013b) using custom macros in IgorPro7.0 software (WaveMetrics).

155 Following physiological recordings, slices were fixed in $0.2 \mathrm{mM}$ phosphate buffer containing $4 \%$

156 paraformaldehyde at $4^{\circ} \mathrm{C}$ for 2 days. Biocytin staining was revealed using Alexa Fluor 594-

157 conjugated streptavidin (Gupta et al. 2012; Swietek et al. 2016).

158 Morphometry and hierarchical cluster analysis. Sections were visualized and imaged using a

159 Zeiss LSM 510 confocal microscope with a 20X, 0.5 NA objective. Cells in which the dendritic

160 trunks appeared severed at the slice surface were excluded from morphometric analysis. Cell

161 reconstructions from confocal image stacks were performed using the directional kernels user-

162 guided reconstruction algorithm in Neurolucida 360 (MBF Bioscience) followed by manual

163 correction and validation in 3D. About 10-20 percent of each dendritic arbor was reconstructed

164 manually. Neurolucida Explorer (MBF Biosciences) was used to extract non-nominal or non-

165 ordinal somatodendritic morphological quantitative parameters (defined in Supplementary

166 Tables 1 \& 2) for use in statistical comparisons and hierarchical cluster analysis. A total of 42 
167 projection neurons in which the dendritic arbors were fully reconstructed were analyzed. The

168 software failed to quantify a subset of somatic parameters in 6 cells, and these cells were 169 excluded from cluster analysis.

170 Data were tested for uniform distribution and each quantified variable was fit to the sum of two 171 or more Gaussian functions and quality of fit determined using maximum likelihood analysis 172 (MLA; v2test) to assess normal distribution of parameters within each cell type. Variables with a 173 nonuniform distribution were used for subsequent cluster analysis. A total of 42 somato-dendritic 174 parameters (Supplementary Tables 1 \& 2) from 36 morphologically reconstructed neurons, 175 including both features measured in Neurolucida Explorer from the 3D reconstructions and 176 parameters measured manually in 2D rendering (Neurolucida 360, MicroBrightfield) were 177 analyzed. Unsupervised clustering and principal component analyses of morphological properties 178 were conducted within R version 3.5.0, using R package Cluster, FactoMineR, and factoextra by 179 an investigator (Y-J C) blinded to cell types and age groups. Hierarchical clustering on the 180 selected principal components (PCs) was performed using Ward's Criterion with Euclidean 181 distance to generate the dendrogram. The clustering partition was obtained from hierarchical 182 clustering and improved with K-means method (Husson et al. 2010). Summary of morphological 183 data and statistical analysis of morphometric parameters are presented in Supplementary Tables 1843 and 4, respectively.

185 Analysis and Statistics: Individual spontaneous inhibitory postsynaptic currents (sIPSCs) were 186 detected using custom software in Igor-Pro7.0 (Gupta et al. 2012). The investigator (AG) was 187 blinded to cell type during analysis. Events were visualized, and any "noise" that spuriously met 188 trigger specifications was rejected. Cumulative probability plots of sIPSC amplitude and 189 frequency were constructed using IgorPro by pooling an equal number of sIPSCs from each cell. 
190 Kinetics and charge transfer were calculated from the averaged trace of all accepted sIPSC

191 events. Rise time was measured as the time for amplitude to change from 20 to $80 \%$ of peak.

192 Amplitude weighted $\tau_{\text {decay }}$ was calculated from a two-exponential fit to the IPSC decay. sIPSC

193 charge transfer was calculated as the area under the curve of the baseline adjusted average sIPSC

194 trace. The summed sIPSC charge transfer over one second was calculated as the product of the

195 sIPSC charge transfer and sIPSC frequency for each cell. $\mathrm{R}_{\mathrm{in}}$ was calculated as the slope of the

196 current voltage plots with voltage response (averaged over the last $400 \mathrm{~ms}$ ) to one second current

197 injections from $-200 \mathrm{pA}$ to $-40 \mathrm{pA}$ (in $40 \mathrm{pA}$ steps) in each cell. Membrane time constant

198 ( $\left.\tau_{\text {membrane }}\right)$ was obtained from a single exponential fit to the voltage response to a $-200 \mathrm{pA}$ current

199 injection. Data are shown as mean \pm SEM (standard error of the mean) or median and interquartile

200 range (IQR) where appropriate and presented in Supplementary Table 5. Kruskal-Wallis One

201 Way Analysis of Variance on ranks was conducted on data which failed Shapiro Wilk test for

202 normalcy or equal variance test. Two-way ANOVA (TW-ANOVA, SigmaPlot 12.3) and

203 Bonferroni correction were used to test for statistical differences in tonic GABA currents.

204 Summary data and details of the statistical tests used are included in Supplementary Tables 5-8.

205 Sample sizes were not predetermined and conformed with those employed in the field.

206 Significance was set to $\mathrm{p}<0.05$, subject to appropriate Bonferroni correction. All custom macros

207 for analysis and data sets will be made available on request upon publication of the study. 


\section{Results}

211 Somato-dendritic characteristics distinguish SGCs from GCs throughout postnatal

\section{2 development.}

213 To examine whether SGCs are structurally distinct from GCs throughout postnatal development,

214 we undertook electrophysiological recordings from putative GCs and SGCs in the GCL and IML

215 respectively, and filled cells with biocytin to recover their morphology. As illustrated by

216 morphological reconstructions obtained from confocal images of biocytin filled neurons (Fig. 1),

217 putative GCs exhibit compact molecular layer dendritic arbors while putative SGCs recorded in

218 the IML consistently exhibited wider dendritic spread. These differences in dendritic spread are

219 maintained throughout the three developmental stages tested. Additionally, the X-Y plane

220 projection of the reconstructed neurons revealed the characteristic crescent shaped somata of

221 SGCs. Interestingly, the arborization in the z plane was not different between GCs and SGCs (in

$222 \mu \mathrm{m}, \mathrm{GC}: 73.6 \pm 8.7, \mathrm{n}=15$; SGC: $69.7 \pm 9.6, \mathrm{n}=18, \mathrm{p}>0.05$ by Student's $t$ test). Additionally, the $\mathrm{z}$

223 plane span of SGCs was lower than their X-Y span indicating that SGCs have a flattened

224 crescentic profile. less than the slice thickness. Figure 1 illustrates pseudo color rendering of

225 dendritic arbors with segments assigned on the basis of branch order in 3D reconstructions in

226 Neurolucida 360. Color coding of the dendritic segments revealed that, in addition to differences

227 in dendritic span, SGCs differed from GCs in the extent to which individual dendritic trees

228 branched and the number of segments in different dendritic orders. Moreover, reconstructions

229 suggest that GCs have a dense distribution of dendritic arbors in a compact volume while SGCs

230 appear to have sparsely distributed dendrites in a larger volume. Thus, while 3D reconstructions

231 demonstrate that SGCs maintain a qualitative pattern of dendritic arborization distinct from GCs 
232 at all postnatal ages examined, they reveal additional morphometric features which differ

233 between cell types indicating a need for comprehensive unsupervised quantitative analysis of

234 dendritic structure.

235 To determine if somato-dendritic parameters distinguish GCs from SGCs through development,

236 we undertook the first unsupervised classification of dentate projection neurons on the basis of

237 morphometry. Projection neurons were identified based on the presence of somata in the GCL

238 (putative GCs) or IML (putative SGCs), axons with boutons entering the hilus and targeting CA3

239 (Supplementary Figure 1). As illustrated by the schematic of the somatic location of recorded

240 cells (Supplementary Figure 2), GCs were recorded from the entire extent of the GCL. We find

241 that both SGCs and GCs, have a high density of dendritic spines (Supplementary Figure 1),

242 which we used as a criterion to distinguish projection neurons from local interneurons when the

243 axon was not fully recovered. Morphometric parameters of the cells reconstructed in 3D were

244 obtained from automated algorithms in Neurolucida 360 (definitions are included in

245 Supplementary Tables 1 and 2). Principal Component Analysis (PCA) of 42 distinct

246 morphometric parameters from 36 cells revealed a relatively high dimensional structure. The

247 first three principal components (PCs) explained about $46 \%$ of the total variance in the data,

248 while the first seven components retained over $85 \%$ of the variance (Supplementary Figure 3).

249 PCA analysis of individual cells was projected on to the first three principal components and

250 visualized in 3D representation with confidence interval (CI) ellipsoid set to 0.95, which

251 suggested that the cells likely segregate by cell type (Fig. 2A) rather than developmental age of

252 the rat (Fig. 2B). 
253 Interestingly, although categorical variables including soma location were not included as a

254 parameters for unsupervised analysis, clustering by PCA matched that of the investigator (Fig.

255 2). A total of 36 dentate projection neurons (16 cells in infant, 10 cells in adolescent and 10 cells

256 in adult), recovered based on the quantitative morphological features, were included in the

257 clustering analysis. A hybrid approach of hierarchical clustering on principal components

258 (HCPC) which helps in de-noising multidimensional dataset was adopted (Husson et al. 2010).

259 HCPC on the first seven components suggested two cluster partitioning groups (Fig 2C).

260 Opening of the blinding revealed that the two putative clusters demonstrated a tendency for cells

261 to cluster by putative cell-type. Cluster 1 included 16 cells classified as GCs and five classified

262 as an SGC by the investigator. Clusters 2 consisted entirely of cell classified as SGCs by the

263 investigator (A.G).

\section{Identity of principal morphometric parameters that best represent the PCs}

265 The PCs were examined further to identify the top five morphological variables which best

266 represent the PC ( $\cos 2 \geq 0.7)$ and the morphological features that contributed to PC variability

267 (Supplementary Figure 3). The number of first, second, and third order dendritic segments (note:

268 number of first order dendritic segments equals number of primary dendrites), number of second

269 order nodes and soma width where the top five morphological features that best represented the

270 PCs while dendritic area and volume contributed most to variability (Supplementary Figure 3).

271 Although the dendritic span and angle, used by the experimenter for classification (Gupta et al.

272 2012; Williams et al. 2007), contributed to the PC, morphology-based clustering revealed

273 additional salient parameters including number of primary dendrites and dendritic segmentation

274 which differed between clusters. Interestingly, one of the cells classified as SGCs by the 
275 investigator which clustered with putative GCs by unsupervised clustering was located in the

276 IML (a feature that was not included in the cluster analysis), had a wide dendritic angle, but had

277 two dendrites, with complex pattern of branching, typically seen in GCs, which could have

278 driven the clustering with GCs (Supplementary Figure 2D $\mathrm{D}_{7}$ ). Importantly, comparison of cell

279 classification based on the unsupervised approach to that of the investigator (A.G.) confirmed

280 that the investigator and PCA-based classifier agreed on the "grouping" of >85\% (31 of 36) of

281 cells examined. These findings confirm that GCs and SGCs are structurally distinct and

282 demonstrate that the investigator can reliably discriminate the cell-types.

283 Developmental changes in somato-dendritic morphology of dentate projection neurons

284 Using the investigator-assigned classifications, we next examined which specific morphological

285 parameters showed cell-type and developmental differences. First, we focused on the parameters

286 that contributed to PCs underlying cell classification. As predicted based on the PCA, the

287 number of primary dendrites (first order segments), second order segments and nodes were

288 different between SGCs and GCs, with SGCs having significantly more dendritic segments in the

289 first three orders of dendrites (Fig. 3A, Supplementary Figure 4 \& Supplementary Tables $3 \& 4$ ).

290 However, the effect of age on the number of segments in each order was not statistically

291 significant (Supplementary Figure 4A\&B, Supplementary Tables 3 \& 4). Similarly, soma width,

292 soma aspect ratio and dendritic angle were greater in SGCs and failed to show age related

293 changes (by TW-ANOVA; Fig. 3B\&C, and Supplementary Tables 3 \& 4). Thus, these

294 parameters are ideally suited to distinguish cell-types regardless of age. In contrast, dendritic

295 length showed a significant effect of age yet was not different between cell types (Fig. 3D, and

296 Supplementary Tables 3 \& 4). Of note, SGCs had significantly lower dendritic complexity than 
297 GCs and failed to show the age-dependent increase in complexity observed in GCs (Fig. 3E, and

298 Supplementary Tables $3 \& 4$ ). Additional parameters that reflected 3D dendritic structure

299 including convex hull surface area showed significant differences between cell-type and with age

300 (Fig. 3F, Supplementary Tables 3 \& 4). The convex hull surface area increased with

301 development from infancy to adolescence and remained at adolescent levels in the adult (Fig. 3F,

302 and Supplementary Tables 3 \& 4) and appeared to contribute substantially to variability in the

303 PCA analysis of pooled morphometric dataset (Supplementary Figure 3B). Finally, certain

304 summed dendritic parameters including total numbers of dendritic terminals (ends), nodes, and

305 segments showed neither cell-type nor age related differences (Supplementary Figure 4D-F,

306 Supplementary Tables 3 \& 4).

\section{Developmental changes in spontaneous synaptic inhibition to GCs and SGCs}

309 We previously demonstrated that SGCs from adolescent rats receive a greater frequency of

310 action-potential driven sIPSCs than GCs from age-matched rats (Gupta et al. 2012). To

311 determine if SGC inhibitory drive changes through postnatal development, we recorded sIPSCs

312 from SGCs and GCs at three developmental stages. As illustrated in Fig. 4, SGCs consistently

313 showed a higher frequency of sIPSCs compared to GCs from age matched rats. Both cell-types

314 showed changes in sIPSC frequency with age. The frequency of sIPSCs in GCs increased from

315 infancy through adolescence, peaked at adolescence and showed a slight, yet significant decrease

316 in adults. (Fig. 4D, Supplementary Figure 5 and Supplementary Tables 5 \& 6). Despite the

317 decrease from adolescence to adulthood, the sIPSC frequency in GCs from adults was higher

318 than that in infancy. SGCs, on the other hand, showed a distinct peak in sIPSC frequency during 
319 adolescence with a significant reduction in frequency in adults, back to the levels observed in

320 infancy (Fig. 4D, Supplementary Figure 5, and Supplementary Tables 5 \& 6). Thus, despite

321 being consistently higher than in GCs, sIPSC frequency in SGCs appears to show a specific and

322 transient enhancement during adolescence.

323 Unlike sIPSC frequency, SGC sIPSC amplitude did not differ from age-matched GCs in infant

324 and adolescent rats (Fig. 5C and Supplementary Tables 5 \& 6), consistent with our earlier reports

325 in adolescent rats (Gupta et al. 2012). However, in adult rats, sIPSC amplitude in GCs was larger

326 than in SGCs (Fig. 5C). In GCs, sIPSC amplitude decreased significantly from infancy to

327 adolescence and remained constant thereafter with differences in sIPSC between adolescence

328 and adulthood not reaching statistical significance (Fig. 5C, Supplementary Tables 5 \& 6). sIPSC

329 amplitude in SGCs decreased through postnatal development with a significant reduction from

330 infancy to adolescence, and a further decline from adolescence to adulthood (Fig. 5C,

331 Supplementary Tables 5 \& 6).

332 Since distal inhibitory inputs can attenuate to a smaller amplitude at the soma (Soltesz et al.

333 1995), we sought to assess whether changes in the proportion of proximal versus distal dendritic

334 synaptic inputs could contribute to developmental change in GC and SGC sIPSC amplitude. To

335 determine if there is a systematic change in the amplitude of sIPSCs with age, we assigned

336 sIPSCs to two groups based on their amplitudes: large and putative proximal and perisomatic

$337(>50 \mathrm{pA})$, and small $(<50 \mathrm{pA})$, potentially dendritic and calculated the proportion of these events

338 classes in GCs and SGCs during development. Consistent with the developmental increase in

339 dendritic length (Fig.3D), the proportion of large amplitude, presumed perisomatic events

340 (>50pA) were highest in infants and reduced progressively with age while the smaller amplitude 
341 events $<50 \mathrm{pA}$ events increased with age in both the cell types (Supplementary Figure 6). These

342 findings suggest that developmental increase in dendritic length may contribute to decline in

343 sIPSC amplitude over age in both cell types.

344 Systematic analysis of sIPSC kinetics identified an overall decline in $\tau_{\text {decay-WT }}$ in GCs with a 345 significant reduction from infant to adolescent rats (Fig. 5D, Supplementary Table 5 \& 7).

346 Similarly, 20-80 rise time in GCs showed an overall decline with age with a significant reduction

347 from infancy to adulthood (Fig. 5E, Supplementary Table 5 \& 7). In contrast, both sIPSC 20-80

348 rise time and $\tau_{\text {decay-WT }}$ increased from infancy through adulthood in SGCs demonstrating a

349 divergence in the effect of development on sIPSC kinetics between GCs and SGCs. Since the

350 developmental changes in both sIPSC rise and $\tau_{\text {decay-WT }}$ showed parallel trends within a given cell

351 type while differing between cell types, we evaluated whether developmental changes in cellular

352 passive membrane parameters may underlie these changes. Consistent with the changes observed

353 in sIPSC kinetics in GCs, both $\mathrm{R}_{\text {in }}$ and membrane time constant ( $\left.\tau_{\text {membrane }}\right)$ trended to decline

354 from infancy through adulthood with the decrease in $\mathrm{R}_{\mathrm{in}}$ reaching statistical significance

355 (Supplementary Fig. 7, Supplementary Tables $5 \&$ 8). As with sIPSC kinetics in SGCs, both

$356 \mathrm{R}_{\text {in }}$ and $\tau_{\text {membrane }}$ increased significantly from infancy through adulthood (Supplementary Fig. 7,

357 Supplementary Tables 8). While direct correlation of the sIPSC kinetics with $\tau_{\text {membrane was not }}$

358 feasible due to use of different cohorts of recordings for sIPSC and passive parameter data, the

359 results suggest that developmental changes in membrane passive properties could, among other

360 factors, contribute to shift in sIPSC kinetics in cell types with postnatal development. Moreover,

361 the data demonstrate that postnatal development has an opposite effect on $\mathrm{R}_{\mathrm{in}}$ in GCs and SGCs.

$362 \mathrm{R}_{\mathrm{in}}$ in SGCs was lower than in GCs in infant and adolescent rats, a feature that has been used to

363 distinguish the cell types (Erwin et al. 2020). However, in adult rats, $\mathrm{R}_{\text {in }}$ in SGCs was 
364 significantly higher than age matched GCs (Supplementary Fig. 7, Supplementary Tables 5 \& 8)

365 revealing that a lower $\mathrm{R}_{\text {in }}$ cannot consistently distinguish SGCs from GCs across all

366 developmental time points.

367 Given the developmental and cell-specific changes in sIPSC peak amplitude and kinetics, we 368 examined whether GCs and SGCs showed changes in sIPSC charge transfer during postnatal 369 development. Consistent with changes in peak amplitude, sIPSC charge transfer declined with

370 development in both cell types (Fig. 5F, Supplementary Tables 5 \& 7). Despite the changes in

371 kinetics, the net charge transfer was not different between GCs and SGCs from age-matched rats

372 (Fig. 5F, Supplementary Tables 5 \& 6). To comprehensively assess spontaneous synaptic

373 inhibition in GCs and SGCs during development in a manner that would include divergent

374 changes in frequency amplitude and kinetics between cell-types and during development, we

375 estimated the cumulative sIPSC charge transfer over one second as a product of the sIPSC

376 frequency and charge transfer for each cell. Interestingly, the cumulative sIPSC charge transfer

377 over one second was maximum during infancy and declined with development in both GCs and

378 SGCs (Supplementary Fig. 5C, Supplementary Table 5 \& 7). Moreover, the cumulative sIPSC

379 charge transfer over one second was not different between cell-types at any developmental stage

380 examined (Supplementary Fig. 5C, Supplementary Table 5 \& 7).

381 Extrasynaptic GABA currents in SGCs peak during adolescence

382 Apart from GABAergic synaptic inputs, dentate GCs are known to express extra- and peri383 synaptic $\mathrm{GABA}_{\mathrm{A}}$ receptors that contribute to steady-state tonic GABA currents (Stell et al. 384 2003). We previously demonstrated the presence of tonic GABA currents in SGCs and identified 385 that the amplitude of tonic GABA current in SGCs was greater than in age-matched adolescent 
386 GCs (Gupta et al. 2012). Here we find that although tonic GABA current amplitude in SGCs,

387 measured as the baseline currents blocked by a saturating concentration of $\mathrm{GABA}_{\mathrm{A}}$ receptor

388 antagonists, was significantly greater than in GCs during adolescence, tonic $\mathrm{GABA}_{\mathrm{A}}$ current

389 amplitude was not different between GCs and SGCs during infancy or adulthood (Fig. 6). Both

390 GCs and SGCs showed a significant increase in tonic GABA currents from infancy to

391 adolescence which returned back to pre-adolescent levels in adults (Fig. 6 and Supplementary

392 Tables 5 \& 6).

393

394 


\section{Discussion:}

396 Contemporary literature on the dentate gyrus describes the projection neurons as a largely

397 homogeneous population of GCs with limited diversity (Kesner 2018). Since GCs are a unique

398 subset of neurons that undergo neurogenesis and maturation through adulthood, structural and

399 functional diversity in GCs is largely attributed to the maturation state of neurons within the

400 circuit (Toda and Gage 2018). At any given postnatal developmental stage of the animal,

401 immature GCs tend to be located closer to the hilar border of the cell layer, have less elaborate

402 dendritic structures and are functionally more excitable than their mature counterparts (Kerloch

403 et al. 2018; Overstreet-Wadiche and Westbrook 2006). However, emerging recognition of a

404 structurally and functionally distinct subset of dentate projection neurons challenges the

405 prevailing view that dentate projection neurons are a homogenous class of cells distinguished by

406 developmental stages (Gupta et al. 2012; Save et al. 2018; Williams et al. 2007). SGCs, which

407 have been characterized in the IML of the dentate gyrus, differ from GCs in their expansive

408 dendritic arbors and show more sustained firing activity (Larimer and Strowbridge 2010;

409 Williams et al. 2007). These and other features, including their distinct window of embryonic

410 day 12-15 for SGC development (Save et al. 2018), enhanced excitatory drive (Larimer and

411 Strowbridge 2010), and distinct synaptic and extrasynaptic inhibitory currents (Gupta et al. 2012)

412 indicate that SGCs are distinct from GCs. Although SGCs have been proposed to sculpt feedback

413 inhibition of GCs, gate dentate activity, and contribute to memory processing (Larimer and

414 Strowbridge 2010; Walker et al. 2010), there is limited information on the structural and

415 physiological differences between GCs and SGCs across postnatal development of the animal.

416 This information is needed to determine which features consistently and reliably distinguish the

417 cell types at all age groups. Our detailed and objective morphometric analysis conducted at three 
418 distinct developmental stages, namely, infant, adolescent, and adult age groups demonstrates that

419 clustering dentate projection neurons based on somato-dendritic morphology distinguishes them

420 into different "subtypes" corresponding to SGCs and GCs. Additionally, SGCs consistently

421 maintained higher frequency of inhibitory synaptic inputs than GCs at all ages. Our results

422 demonstrate that although both GCs and SGCs exhibit peaks in frequency of synaptic inhibitory

423 inputs and magnitude of tonic GABA currents during adolescence, these parameters were

424 significantly higher in SGCs than in GCs at this time point. However, the peak sIPSC amplitude

425 and cumulative charge transfer were highest in infants, decreased with development and were not

426 different between cell types. These findings demonstrate that SGCs are a structurally and

427 functionally distinct subtype of dentate projection neurons which is in keeping with the emerging

428 recognition of subpopulations among hippocampal and entorhinal projection neurons (Pilli et al.

429 2012; Soltesz and Losonczy 2018). Moreover, the significantly heightened tonic inhibition in

430 SGCs during adolescence suggests that SGC activity could be more strongly modulated than

431 GCs by a variety of neuroactive compounds including alcohol and neurosteroids, which

432 selectively augment extrasynaptic GABA currents (Maguire and Mody 2009; Mody et al. 2007).

433 Future studies examining effects of alcohol and neurosteroids on SGC function during

434 adolescence coupled with analysis of developmental changes in excitatory drive and active

435 properties of GCs and SGCs can provide a more comprehensive understanding of the network 436 role of SGCs.

437 In addition to using unbiased approaches to classify SGCs as a distinct neuronal class, our data 438 identify key age-invariant features to distinguish the SGCs from GCs. We find that the number 439 of primary dendrites, dendritic angle, soma width and soma ratio are significantly higher in 440 SGCs and can be used to categorize SGCs and GCs. In particular, the multiple primary dendrites 
441 observed in SGCs stands in stark contrast to the typical one to two apical dendrites observed in

442 granule cell reconstructions (Thind et al. 2008). Indeed, the striking $>85 \%$ correspondence

443 between the unsupervised clustering and investigator assigned clustering likely stems from the

444 investigator's use of dendritic angle, soma width, which are significant contributors to the first

445 PC, in addition to soma location to classify cell types. Dendritic length, on the other hand,

446 increased during development but was not different between cell types indicating that processes

447 reflecting developmental maturation are common to the cell types. Consistent with the presence

448 of larger dendritic angle, the convex hull 3D volume and 3D surface area of SGCs were greater

449 than that of GCs. However, there was also a developmental increase in these parameters from

450 infancy to adolescence and a further stabilization into adulthood in both cell types, likely

451 reflecting the developmental increase in hippocampal volume. While extensive precautions were

452 taken to exclude cells with severed dendrites from morphometric analysis, it remains possible

453 that the fills in slices may have missed some dendritic branches. However, our finding that the $\mathrm{z}$

454 axis thickness is not different between GCs and SGCs and is considerably lower than both slice

455 thickness and X-Y span indicate that dendritic arbors of both cell types are likely fully

456 represented within the slice. Interestingly, although the number of

457 terminal nodes was not different, the dendritic complexity was significantly lower in SGCs than

458 in GCs demonstrating differences in branching patterns. Difference in branching patterns

459 including dendritic complexity can impact neuronal firing and intracellular signaling, as has been

460 demonstrated in modeling studies ( $\mathrm{Li}$ et al. 2015; van der Velden et al. 2012; van Elburg and van

461 Ooyen 2010). Whether dendritic morphology can account for differences in intrinsic physiology

462 between the dentate projection neuron types (Gupta et al. 2012; Save et al. 2018; Williams et al.

463 2007) remains to be examined. The other aspect where distinguishing cell type based on 
464 morphology becomes critical is in disease. Dentate granule cells are known to undergo changes

465 in dendritic structure including alterations in complexity under physiological conditions and in

466 trauma and neurodegenerative diseases (Llorens-Martin et al. 2015; Redila and Christie 2006;

467 Villasana et al. 2015). The ability to distinguish GCs and SGCs across multiple developmental

468 stages would be crucial to quantifying and interpreting changes in morphology in trauma,

469 epilepsy, and neurodegenerative disease.

470 While there are clear differences in the structure, SGCs and GCs share several characteristics.

471 Both SGCs and GCs are projection neurons, with dendrites in the dentate molecular layer and

472 axons projecting to hippocampal CA3 (Gupta et al. 2012; Save et al. 2018; Williams et al. 2007).

473 Several dendritic parameters including total numbers of dendritic segments, nodes, terminals,

474 and dendritic tortuosity (Supplementary Table 3) showed neither cell type nor age-related

475 differences. Similarly, SGCs, like GCs, have dendritic spines which can aid in distinguishing

476 them from inhibitory neurons. Additionally, hilar axon collaterals of SGCs have "mossy fiber

477 boutons" typically attributed to GCs (Supplementary Fig. 1 and Save et al. 2018). Notably, in

478 pilot clustering analysis which included a few molecular layer inhibitory neurons, the

479 interneurons clustered on a different branch of the dendrogram than SGCs and GCs (data not

480 shown). The similarities between GCs and SGCs are not surprising, since we previously

481 identified that SGCs express the Prospero homeobox protein 1 (Prox1) present in GCs indicating

482 a shared lineage (Gupta et al. 2012). This shared lineage was confirmed by a recent study which

483 identified a narrow developmental window of embryonic day 12-15 during which SGCs are

484 produced from the same precursor niche as GCs (Save et al. 2018). Yet, the morphology of

485 embryonically labeled SGCs were distinct from that of GCs labeled on the same day (Save et al.

486 2018), demonstrating that they are a distinct population of cells rather than a cohort of GCs with 
487 a different maturation state. In this context, studies examining dendritic properties of GCs with

488 somata located in the outer third of the molecular layer have consistently reported wider

489 dendritic fields and distinct dendritic arbors consistent with the possibility that the outer third of

490 the granule cell layer may consist of a mixed population of GCs and SGCs (Green and Juraska

491 1985; Kerloch et al. 2018; Sun et al. 2013). Whether specific genetic, molecular or

492 developmental cues guide the development of SGCs during neurogenesis or whether local

493 molecular and spatial factors in the dentate GCL-molecular layer border contribute to elaboration

494 of distinct dendritic arbors remains to be determined (Hatami et al. 2018; Lefebvre et al. 2015).

495 Similarly, whether SGCs and GCs have comparable dendritic length distribution within the

496 distinct axonal projection zones, namely, commissural/associational pathway in the IML, medial

497 perforant path in the middle molecular layer and lateral perforant path in the outer molecular

498 layer was outside the scope of the current study and merits further analysis. Although the

499 mechanisms underlying specification of SGCs as a distinct population and the layer specific

500 inputs to SGCs need further investigation, our objective unsupervised morphometric analysis

501 identifies the key somato-dendritic structural features that distinguish SGCs from GCs through

502 postnatal development.

503 A defining feature of the dentate gyrus is the presence of heavy inhibitory regulation (Coulter

504 and Carlson 2007). Dentate GCs receive synaptic inhibition from a diverse population of neurons

505 and are also under steady-state, tonic extrasynaptic inhibition (Coulter and Carlson 2007; Ewell

506 and Jones 2010; Harney and Jones 2002; Stell et al. 2003). In an earlier study, we identified that

507 SGCs are under stronger inhibitory regulation than GCs with higher frequency of inhibitory

508 synaptic currents and greater amplitude of tonic GABA currents (Gupta et al. 2012). Here, we

509 find that SGCs continue to receive greater spontaneous synaptic inhibitory events than GCs 
510 through postnatal development. This contrasts with the lack of difference in the frequency of

511 spontaneous IPSCs in mature granule cells regardless of whether they were born embryonically

512 or in adults (Laplagne et al. 2007) in spite of the complex and extended maturation of inhibitory

513 synapses on to adult born granule cells (Dieni et al. 2013; Groisman et al. 2020). Interestingly,

514 while the frequency of sIPSCs peaked during adolescence in both cell types, the amplitude

515 decreases progressively with development. Additionally, while synaptic membrane kinetics

516 decreased with development in GCs, they increased with development of SGCs. The combined

517 effect of cell specific and developmental changes resulted in an overall decrease in cumulative

518 synaptic inhibitory charge transfer from infancy to adulthood while maintaining similar charge

519 transfer between age-matched GCs and SGCs. In addition to developmental changes in $\tau_{\text {membrane }}$

520 which could contribute to cell-specific regulation of synaptic kinetics, the roles of changes in

521 GABA receptor subunits, dendritic pruning and synaptic distribution need to be considered in

522 future works. Although lower $\mathrm{R}_{\text {in }}$ in SGCs has been considered a defining distinction from GCs

523 (Erwin et al. 2020; Gupta et al. 2012; Williams et al. 2007), our demonstration that SGCs in

524 adult rats have higher $\mathrm{R}_{\text {in }}$ than in GCs suggests that $\mathrm{R}_{\text {in }}$ may not be an age-invariant feature for

525 cell classification.

526 Can dendritic structural features explain the cell-type specific and developmental differences in

527 sIPSC frequency in dentate projection neurons? The consistently higher sIPSC frequency in

528 SGCs is surprising as the dendritic lengths and location are not different between GCs and SGCs

529 (Fig. 3). The wider dendritic distribution of SGCs, also reflected in the greater convex hull 3D

530 volume, could allow for inputs from a larger group of inhibitory neurons to impinge on SGC

531 dendrites, while GCs with their compact dendritic distribution may receive fewer inputs. It is

532 also possible that the relatively early embryonic development of SGCs increases the chance of 
533 SGCs to receive more synaptic inputs compared to GCs which develop later into adulthood.

534 However, since both dendritic length and convex hull 3D volume increase with postnatal

535 development of both cells, changes in dendritic morphology and embryonic development are

536 unlikely to account for the peak in sIPSC frequency in adolescence followed by decline in adults.

537 The possibility of developmental increase in synapses from infancy to adolescence followed by

538 pruning or synapse elimination into adulthood (Riccomagno and Kolodkin 2015; Tran et al.

539 2009) may be considered in future works. Although multiple precautions were taken to maintain

540 tissue health in slices from adult rats, it is possible that differences in interneuron viability in

541 slices from adolescent versus adult rats also contributed to decline in IPSC frequency with age.

542 The progressive decrease in sIPSC amplitude with development in both SGCs and GCs could

543 reflect the increase in dendritic length with development. Consistent with this proposal, the

544 proportion of large amplitude events decrease progressively with development in both cell types

545 (Supplementary Figure 6).

546 In parallel, the amplitude of extrasynaptic GABA currents in SGCs peaked during adolescence.

547 Similarly, tonic GABA currents in GCs showed a peak during adolescence confirming the

548 developmental increase reported in previous studies (Holter et al. 2010). Increase in expression

549 of the extrasynaptic $\mathrm{GABA}_{\mathrm{A}} \mathrm{R} \delta$ subunit in both GCs and SGCs (Gupta et al. 2012; Maguire and

550 Mody 2009) is likely to mediate the increase in tonic GABA currents from infancy to

551 adolescence. Additionally, changes in synaptically released GABA accompanying changes in

552 sIPSC frequency, reported here, could contribute to the peak in extrasynaptic GABA exhibited in

553 adolescence. An interesting feature of tonic GABA currents mediated by $\mathrm{GABA}_{\mathrm{A}} \mathrm{R} \delta$ subunit is

554 their robust enhancement by neurosteroids, raising the possibility that increases in ambient

555 neurosteroids during adolescence (Harden and MacLusky 2004; Maguire and Mody 2009) could 
augment tonic GABA currents. Since tonic GABA currents mediated by $\mathrm{GABA}_{\mathrm{A}} \mathrm{R} \delta$ subunits are

557 exquisitely sensitive to ethanol (Mody et al. 2007), the greater magnitude of tonic GABA

558 currents in SGCs during adolescence is likely to render SGCs vulnerable to ethanol modulation

559 and impact their role in dentate processing. Indeed, behavioral deficits following alcohol

560 administration have been shown to be particularly accentuated during adolescence (Lacaille et al.

561 2015; Spanos et al. 2012). It would be important for future studies to ascertain how the

562 differences in basal synaptic inhibition impact the recruitment and circuit function of SGCs and

563 GCs during network activity and with development. Since basal inhibition in the dentate gyrus

564 regulates synaptic plasticity and pattern separation at the level of the circuit (Dengler and Coulter

565 2016; Madar et al. 2019), and SGCs have been proposed to contribute to dentate pattern

566 separation (Larimer and Strowbridge 2010), developmental changes in GC and SGC inhibition

567 are likely to influence memory and cognitive performance.

568 Together, the structural and functional data identify SGCs as a cell type which differs from GCs

569 in somato-dendritic structure and developmental plasticity of inhibitory inputs, extrasynaptic

570 inhibition and membrane kinetics. Our data demonstrate that SGCs have heightened

571 extrasynaptic inhibition during adolescence which would make them susceptible to endogenous

572 and exogenous modulation of activity levels during adolescence. Notably, our results delineate

573 salient structural features that enable anatomical identification of this subpopulation of dentate

574 projection neurons. The novel data defining the structural features of SGCs will allow for future

575 targeted analysis of their molecular profile and microcircuit connectivity to better understand

576 their role in circuit function and behaviors. In conclusion, the fundamental characterization of

577 SGCs presented here will support incorporation of SGCs into current models of the dentate gyrus

578 and consideration of their role in dentate microcircuit processing in health and disease. 
bioRxiv preprint doi: https://doi.org/10.1101/2019.12.17.880005; this version posted September 15, 2020. The copyright holder for this preprint (which was not certified by peer review) is the author/funder. All rights reserved. No reuse allowed without permission.

Gupta et al, Page 29 of $\mathbf{4 3}$ 


\section{Reference List}

581 Coulter DA, Carlson GC (2007) Functional regulation of the dentate gyrus by GABA-mediated 582 inhibition ProgBrain Res 163:235-243

583 Dengler CG, Coulter DA (2016) Normal and epilepsy-associated pathologic function of the

584 dentate gyrus Prog Brain Res 226:155-178 doi:10.1016/bs.pbr.2016.04.005

585 Dieni CV, Chancey JH, Overstreet-Wadiche LS (2012) Dynamic functions of GABA signaling

586 during granule cell maturation Front Neural Circuits 6:113 doi:10.3389/fncir.2012.00113

587 Dieni CV, Nietz AK, Panichi R, Wadiche JI, Overstreet-Wadiche L (2013) Distinct determinants

588 of sparse activation during granule cell maturation J Neurosci 33:19131-19142

589 doi:10.1523/JNEUROSCI.2289-13.2013

590 Duffy CJ, Rakic P (1983) Differentiation of granule cell dendrites in the dentate gyrus of the

591 rhesus monkey: a quantitative Golgi study J Comp Neurol 214:224-237

592 doi:10.1002/cne.902140210

593 Engel D, Jonas P (2005) Presynaptic action potential amplification by voltage-gated Na+

594 channels in hippocampal mossy fiber boutons Neuron 45:405-417

595 doi:10.1016/j.neuron.2004.12.048

596 Erwin SR, Sun W, Copeland M, Lindo S, Spruston N, Cembrowski MS (2020) A Sparse,

597 Spatially Biased Subtype of Mature Granule Cell Dominates Recruitment in Hippocampal-

598 Associated Behaviors Cell Rep 31:107551 doi:10.1016/j.celrep.2020.107551

599 Ewell LA, Jones MV (2010) Frequency-tuned distribution of inhibition in the dentate gyrus The

600 Journal of neuroscience : the official journal of the Society for Neuroscience 30:12597-12607

601 doi:10.1523/JNEUROSCI.1854-10.2010 
602 Freiman TM, Eismann-Schweimler J, Frotscher M (2011) Granule cell dispersion in temporal

603 lobe epilepsy is associated with changes in dendritic orientation and spine distribution Exp

604 Neurol 229:332-338 doi:10.1016/j.expneurol.2011.02.017

605 Green EJ, Juraska JM (1985) The dendritic morphology of hippocampal dentate granule cells

606 varies with their position in the granule cell layer: a quantitative Golgi study Exp Brain Res

$607 \quad 59: 582-586$

608 Groisman AI, Yang SM, Schinder AF (2020) Differential Coupling of Adult-Born Granule Cells

609 to Parvalbumin and Somatostatin Interneurons Cell Rep 30:202-214 e204

610 doi:10.1016/j.celrep.2019.12.005

611 Gupta A, Elgammal FS, Proddutur A, Shah S, Santhakumar V (2012) Decrease in tonic

612 inhibition contributes to increase in dentate semilunar granule cell excitability after brain injury $\mathbf{J}$

613 Neurosci 32:2523-2537 doi:10.1523/JNEUROSCI.4141-11.2012

614 Harden C, MacLusky NJ (2004) Aromatase inhibition, testosterone, and seizures Epilepsy Behav

615 5:260-263 doi:10.1016/j.yebeh.2003.12.001

616 Harney SC, Jones MV (2002) Pre- and postsynaptic properties of somatic and dendritic

617 inhibition in dentate gyrus Neuropharmacology 43:584-594

618 Hatami M, Conrad S, Naghsh P, Alvarez-Bolado G, Skutella T (2018) Cell-Biological

619 Requirements for the Generation of Dentate Gyrus Granule Neurons Front Cell Neurosci 12:402

620 doi:10.3389/fncel.2018.00402

621 Hollrigel GS, Ross ST, Soltesz I (1998) Temporal patterns and depolarizing actions of

622 spontaneous GABAA receptor activation in granule cells of the early postnatal dentate gyrus $\mathbf{J}$

623 Neurophysiol 80:2340-2351 doi:10.1152/jn.1998.80.5.2340 
624 Holter NI, Zylla MM, Zuber N, Bruehl C, Draguhn A (2010) Tonic GABAergic control of

625 mouse dentate granule cells during postnatal development Eur J Neurosci 32:1300-1309

626 doi:10.1111/j.1460-9568.2010.07331.x

627 Husson F, Josse J, Pages J (2010) Principal component methods - hierarchical clustering -

628 partitional clustering: why would we need to choose for visualizing data?

629 Kerloch T, Clavreul S, Goron A, Abrous DN, Pacary E (2018) Dentate Granule Neurons

630 Generated During Perinatal Life Display Distinct Morphological Features Compared With Later-

631 Born Neurons in the Mouse Hippocampus Cereb Cortex doi:10.1093/cercor/bhy224

632 Kesner RP (2018) An analysis of dentate gyrus function (an update) Behav Brain Res 354:84-91

633 doi:10.1016/j.bbr.2017.07.033

634 Lacaille H, Duterte-Boucher D, Liot D, Vaudry H, Naassila M, Vaudry D (2015) Comparison of

635 the deleterious effects of binge drinking-like alcohol exposure in adolescent and adult mice $\mathrm{J}$

636 Neurochem 132:629-641 doi:10.1111/jnc.13020

637 Laplagne DA, Kamienkowski JE, Esposito MS, Piatti VC, Zhao C, Gage FH, Schinder AF

638 (2007) Similar GABAergic inputs in dentate granule cells born during embryonic and adult

639 neurogenesis Eur J Neurosci 25:2973-2981 doi:10.1111/j.1460-9568.2007.05549.x

640 Larimer P, Strowbridge BW (2010) Representing information in cell assemblies: persistent

641 activity mediated by semilunar granule cells Nat Neurosci 13:213-222 doi:10.1038/nn.2458

642 Lee CY, Liou HH (2013) GABAergic tonic inhibition is regulated by developmental age and

643 epilepsy in the dentate gyrus Neuroreport 24:515-519 doi:10.1097/WNR.0b013e32836205bc

644 Lefebvre JL, Sanes JR, Kay JN (2015) Development of dendritic form and function Annu Rev

645 Cell Dev Biol 31:741-777 doi:10.1146/annurev-cellbio-100913-013020 
646 Li L, Gervasi N, Girault JA (2015) Dendritic geometry shapes neuronal cAMP signalling to the

647 nucleus Nat Commun 6:6319 doi:10.1038/ncomms7319

648 Li Y, Aimone JB, Xu X, Callaway EM, Gage FH (2012) Development of GABAergic inputs

649 controls the contribution of maturing neurons to the adult hippocampal network Proc Natl Acad

650 Sci U S A 109:4290-4295 doi:10.1073/pnas.1120754109

651 Llorens-Martin M, Rabano A, Avila J (2015) The Ever-Changing Morphology of Hippocampal

652 Granule Neurons in Physiology and Pathology Front Neurosci 9:526

653 doi:10.3389/fnins.2015.00526

654 Madar AD, Ewell LA, Jones MV (2019) Temporal pattern separation in hippocampal neurons

655 through multiplexed neural codes PLoS Comput Biol 15:e1006932

656 doi:10.1371/journal.pcbi.1006932

657 Maguire J, Mody I (2009) Steroid hormone fluctuations and GABA(A)R plasticity

658 Psychoneuroendocrinology 34 Suppl 1:S84-90 doi:10.1016/j.psyneuen.2009.06.019

659 Mody I, Glykys J, Wei W (2007) A new meaning for "Gin \& Tonic": tonic inhibition as the

660 target for ethanol action in the brain Alcohol 41:145-153 doi:10.1016/j.alcohol.2007.03.009

661 Overstreet-Wadiche LS, Westbrook GL (2006) Functional maturation of adult-generated granule

662 cells Hippocampus 16:208-215 doi:10.1002/hipo.20152

663 Pilli J, Abbasi S, Richardson M, Kumar SS (2012) Diversity and excitability of deep-layer

664 entorhinal cortical neurons in a model of temporal lobe epilepsy J Neurophysiol 108:1724-1738

665 doi:10.1152/jn.00364.2012

666 Ramón y Cajal S (1953) Histologie du système nerveux de l'homme \& des vertébrés. Éd.

667 franc $\square$ aise rev. \& mise à jour edn. Consejo Superior de Investigaciones Científicas, Instituto

668 Ramón y Cajal, Madrid, 
669 Redila VA, Christie BR (2006) Exercise-induced changes in dendritic structure and complexity

670 in the adult hippocampal dentate gyrus Neuroscience 137:1299-1307

671 doi:10.1016/j.neuroscience.2005.10.050

672 Riccomagno MM, Kolodkin AL (2015) Sculpting neural circuits by axon and dendrite pruning

673 Annu Rev Cell Dev Biol 31:779-805 doi:10.1146/annurev-cellbio-100913-013038

674 Sancho-Bielsa FJ, Navarro-Lopez JD, Alonso-Llosa G, Molowny A, Ponsoda X, Yajeya J,

675 Lopez-Garcia C (2012) Neurons of the dentate molecular layer in the rabbit hippocampus PLoS

676 One 7:e48470 doi:10.1371/journal.pone.0048470

677 Save L, Baude A, Cossart R (2018) Temporal Embryonic Origin Critically Determines Cellular

678 Physiology in the Dentate Gyrus Cereb Cortex doi:10.1093/cercor/bhy132

679 Semple BD, Blomgren K, Gimlin K, Ferriero DM, Noble-Haeusslein LJ (2013) Brain

680 development in rodents and humans: Identifying benchmarks of maturation and vulnerability to

681 injury across species Prog Neurobiol 106-107:1-16 doi:10.1016/j.pneurobio.2013.04.001

682 Sengupta P (2013) The Laboratory Rat: Relating Its Age With Human's Int J Prev Med 4:624-

683630

684 Seress L, Frotscher M (1990) Morphological variability is a characteristic feature of granule cells

685 in the primate fascia dentata: a combined Golgi/electron microscope study J Comp Neurol

$686 \quad 293: 253-267$

687 Soltesz I, Losonczy A (2018) CA1 pyramidal cell diversity enabling parallel information

688 processing in the hippocampus Nat Neurosci 21:484-493 doi:10.1038/s41593-018-0118-0

689 Soltesz I, Smetters DK, Mody I (1995) Tonic inhibition originates from synapses close to the

690 soma Neuron 14:1273-1283 
691 Spanos M, Besheer J, Hodge CW (2012) Increased sensitivity to alcohol induced changes in

692 ERK Map kinase phosphorylation and memory disruption in adolescent as compared to adult

693 C57BL/6J mice Behav Brain Res 230:158-166 doi:10.1016/j.bbr.2012.02.010

694 Stell BM, Brickley SG, Tang CY, Farrant M, Mody I (2003) Neuroactive steroids reduce

695 neuronal excitability by selectively enhancing tonic inhibition mediated by delta subunit-

696 containing GABAA receptors Proc Natl Acad Sci U S A 100:14439-14444

697 doi:10.1073/pnas.2435457100

698 Sun GJ, Sailor KA, Mahmood QA, Chavali N, Christian KM, Song H, Ming GL (2013)

699 Seamless reconstruction of intact adult-born neurons by serial end-block imaging reveals

700 complex axonal guidance and development in the adult hippocampus J Neurosci 33:11400-

$701 \quad 11411$ doi:10.1523/JNEUROSCI.1374-13.2013

702 Swietek B, Gupta A, Proddutur A, Santhakumar V (2016) Immunostaining of Biocytin-filled and

703 Processed Sections for Neurochemical Markers J Vis Exp doi:10.3791/54880

704 Tanaka Y, Tanaka Y, Furuta T, Yanagawa Y, Kaneko T (2008) The effects of cutting solutions

705 on the viability of GABAergic interneurons in cerebral cortical slices of adult mice J Neurosci

706 Methods 171:118-125 doi:10.1016/j.jneumeth.2008.02.021

707 Thind KK, Ribak CE, Buckmaster PS (2008) Synaptic input to dentate granule cell basal

708 dendrites in a rat model of temporal lobe epilepsy J Comp Neurol 509:190-202

709 doi:10.1002/cne.21745

710 Toda T, Gage FH (2018) Review: adult neurogenesis contributes to hippocampal plasticity Cell

711 Tissue Res 373:693-709 doi:10.1007/s00441-017-2735-4

712 Tran TS et al. (2009) Secreted semaphorins control spine distribution and morphogenesis in the

713 postnatal CNS Nature 462:1065-1069 doi:10.1038/nature08628 
714 van der Velden L, van Hooft JA, Chameau P (2012) Altered dendritic complexity affects firing

715 properties of cortical layer 2/3 pyramidal neurons in mice lacking the 5-HT3A receptor $\mathbf{J}$

716 Neurophysiol 108:1521-1528 doi:10.1152/jn.00829.2011

717 van Elburg RA, van Ooyen A (2010) Impact of dendritic size and dendritic topology on burst

718 firing in pyramidal cells PLoS Comput Biol 6:e1000781 doi:10.1371/journal.pcbi.1000781

719 Villasana LE, Kim KN, Westbrook GL, Schnell E (2015) Functional Integration of Adult-Born

720 Hippocampal Neurons after Traumatic Brain Injury(1,2,3) eNeuro 2

721 doi:10.1523/ENEURO.0056-15.2015

722 von Campe G, Spencer DD, de Lanerolle NC (1997) Morphology of dentate granule cells in the

723 human epileptogenic hippocampus Hippocampus 7:472-488 doi:10.1002/(SICI)1098-

724 1063(1997)7:5<472::AID-HIPO4>3.0.CO;2-J

725 Walker MC, Pavlov I, Kullmann DM (2010) A 'sustain pedal' in the hippocampus? Nature

726 neuroscience 13:146-148 doi:10.1038/nn0210-146

727 Williams PA, Larimer P, Gao Y, Strowbridge BW (2007) Semilunar granule cells: glutamatergic

728 neurons in the rat dentate gyrus with axon collaterals in the inner molecular layer J Neurosci

729 27:13756-13761 doi:10.1523/JNEUROSCI.4053-07.2007

730 Yu EP, Dengler CG, Frausto SF, Putt ME, Yue C, Takano H, Coulter DA (2013a) Protracted

731 postnatal development of sparse, specific dentate granule cell activation in the mouse

732 hippocampus J Neurosci 33:2947-2960 doi:10.1523/JNEUROSCI.1868-12.2013

733 Yu J, Proddutur A, Elgammal FS, Ito T, Santhakumar V (2013b) Status epilepticus enhances

734 tonic GABA currents and depolarizes GABA reversal potential in dentate fast-spiking basket

735 cells J Neurophysiol 109:1746-1763 doi:10.1152/jn.00891.2012 
736 Yu J, Proddutur A, Swietek B, Elgammal FS, Santhakumar V (2016) Functional Reduction in

737 Cannabinoid-Sensitive Heterotypic Inhibition of Dentate Basket Cells in Epilepsy: Impact on

738 Network Rhythms Cereb Cortex 26:4229-4314 doi:10.1093/cercor/bhv199 


\section{Figure Legends}

741 Figure 1. Somato-dendritic differences in dentate projection neurons - GCs and SGCs.

742 Representative neuronal reconstructions of GC (1A-F) and SGC (1G-L) showing distinct

743 dendritic arbors from infant (top), adolescent (middle) and adult (bottom) age groups. Note that

744 images are shown in different planes for each age group. The complexity of dendrites is shown

745 in XY planes for GCs and SGCs 'A, B, C' and ' $\mathrm{G}, \mathrm{H}, \mathrm{I}$ ' respectively and perpendicular view is

746 shown in 'D, E, F' for GCs and 'J, K, L' for SGCs. Inset images in the center represent the color

747 coding for dendritic segments from proximal to distal axes. Scale bar: $100 \mu \mathrm{m}$.

748 Figure 2. Hierarchical clustering on principal component reveals two major clusters of

749 dentate projection neurons. (A-B) 3D representation of the principal component analysis of

750 individuals resolved by first three principal components, with confidence interval (CI) ellipsoid

751 set to 0.95 . The plot suggests grouping by cell type (A) but not by age (B). (C) Hierarchical

752 clustering on Principal Components based on 42 morphometric parameters (Supplementary

753 Tables 1 \& 2) was performed using Ward's method with Euclidean distance to generate the

754 dendrogram. Dendrogram classifies neurons into two putative clusters. Cells in which the

755 classifier and experimenter failed to concur on classification are represented by green

756 arrowheads based on investigator assigned classification.

757 Figure 3: Comparison of morphometric parameters between GCs and SGCs at distinct

758 developmental stages. Summary plots of averages no. of primary dendrites (A), soma width (B),

759 dendritic angle (C), total dendritic length (D), dendritic complexity (E), and convex hull surface

760 area $(\mathrm{F})$ of GCs and SGCs at three developmental time points. *, \#, and $\$$ denote $\mathrm{p}<0.05$ for 
761 differences between cell types, in GC across age groups and SGC across age groups, respectively

762 by TW-ANOVA followed by post-hoc pairwise comparison (Supplementary Tables $3 \& 4$ ).

$763 \mathrm{~N}=$ GCs, 6 infant, 6 adolescent and 4 adult and SGCs, 9 infant, 5 adolescent and 6 adult.

Figure 4: Developmental differences in sIPSC frequencies of GCs and SGCs. Representative sIPSC traces in GCs (left, A1, B1, C1) and SGCs (right, A2, B2, C2) in infant, adolescent, and adult age groups. Summary plot of developmental differences in sIPSC frequency between and within GCs and SGCs (D). *, \#, and $\$$ denote $\mathrm{p}<0.05$ for differences between cell types, in GC across age groups and SGC across age groups, respectively by TW-ANOVA followed by posthoc pairwise comparison (Supplementary Tables $5 \& 6$ ). Cumulative probability plots of sIPSCs

771 frequency show differences between cell types at infant (E1), adolescent (E2) and adult (E3) age 772 groups * denotes $\mathrm{p}<0.05$ for differences between cell types by Kruskal-Wallis Test

773 (Supplementary Tables 5 \& 6).

\section{Figure 5: Developmental changes in average sIPSC parameters in GCs and SGCs. Overlay}

775 of representative average sIPSC waveforms from GCs (A) and SGCs (B) in different age

776 groups. Summary plots of sIPSC amplitude (C), weighted $\tau_{\text {decay }}(\mathrm{D}), 20-80$ rise time $(\mathrm{E})$, and

777 charge transfer $(\mathrm{F})$ at three developmental stages in both cell types. *, \#, and $\$$ denote $\mathrm{p}<0.05$ for

778 differences between cell types, in GC across age groups and SGC across age groups, respectively

779 by TW-ANOVA followed by post-hoc pairwise comparison (Supplementary Tables 5 - 8).

780 Figure 6: Extrasynaptic tonic GABAergic currents in SGCs peak during adolescence.

781 Representative baseline current recordings in GCs (left, A1-3) and SGCs (right, B1-3) in infant,

782 adolescent and adult age groups. Tonic GABA current is measured as the difference in baseline 
783 current upon perfusion of the GABA receptor antagonist BMI $(100 \mu \mathrm{M})$. Gaussian fit to the

784 positive half of the baseline current, under basal conditions and in BMI, is illustrated on the right

785 of each trace and was used to quantify tonic GABA. (C) Summary plot showing average tonic

786 GABAergic currents at three distinct time points. *, \#, and $\$$ denote $\mathrm{p}<0.05$ for differences

787 between cell types, in GCs across age groups and in SGCs across age groups, respectively by

788 TW-ANOVA followed by post-hoc pairwise comparison (Supplementary Tables $5 \& 6$ ). 


\section{Supplementary Figure legends}

792 Supplementary Figure 1: Representative images of a GC and SGC. Images of a typical GC

793 (A) and SGC (B) illustrate the somatic location, dendritic arbor, with high density of spines

794 (insets, white arrows) and axons with boutons (white arrow heads) targeting CA3 used by

795 experimenter to classify SGCs and GCs. Scale bar: $100 \mu \mathrm{m}$; Inset scale bar: $20 \mu \mathrm{m}$.

796 Supplementary Figure 2: Distribution of somatic location of cells included in morphometric

797 analysis. A. Schematic of the somatic location in the GCL (green box) and IML (orange box)

798 overlaid on the cluster analysis distribution illustrates SGCs in blue and GCs in green. $\mathrm{B}_{1-3}$ :

799 Montage of biocytin filled images of representative GCs (labeled as $\mathrm{B}_{1-3}$ in A) with GCL

800 demarcated by dotted lined identified based on Prox-1 $\left(\mathrm{B}_{1-2}\right)$ or parvalbumin $\left(\mathrm{B}_{3}\right)$

801 immunostaining. C. Representative SGC reconstruction in IML overlaid on image of the slice.

$802 \mathrm{D}_{1-7}$. Examples of biocytin filled and reconstructed GCs and SGCs labeled as $\mathrm{D}_{1-7}$ in panel A.

803 Note that the SGCs illustrated here clustered with putative GCs and are included in the white

804 dotted area in A. All images were obtained a 20X magnification and reconstructed. Note the

805 difference in soma shape of cells classified as GCs $\left(D_{1-3}\right)$ versus those classified as $\operatorname{SGCs}\left(\mathrm{D}_{4-7}\right)$

806 by investigator. $\mathrm{D}_{7}$. Note the increased dendritic complexity compared to putative SGCs in $\mathrm{D}_{4-6}$.

807 GCL: Granule Cell Layer, IML: Inner Molecular Layer.

808 Supplementary Figure 3: Analysis of Morphological Variables underlying Principal

809 Components. A. Histogram illustrates percentage of information retained by each dimension

810 (principal component, PC). B. Factor maps illustrate the quality of representation of the

811 morphometric variables measured by $\cos 2$ (square cosine, squared coordinates). The darker color 
812 indicates stronger contribution to variability to PC. C. Representation of the top five variables in

813 the first two dimensions..

814 Supplementary Figure 4: Comparison of morphometric parameters between GCs and

815 SGCs at distinct developmental stages. Summary plot of number of second (A) and third (B)

816 order dendritic segments, second order nodes (C), total number of dendritic ends (D), total

817 dendritic nodes (E) and total dendritic segments (F) in GCs and SGCs at the three age groups

818 examined. * denotes $\mathrm{p}<0.05$ for differences between cell types by TW-ANOVA followed by

819 post-hoc pairwise comparison (Supplementary Tables 3 \& 4). N= GCs, 6 infant, 6 adolescent and

8204 adult and SGCs, 9 infant, 5 adolescent and 6 adult.

822 Supplementary Figure 5: Cumulative plots of sIPSC frequency in GCs and SGCs through

823 development. Cumulative plots of sIPSC frequency comparing three age groups * denotes

$824 \mathrm{p}<0.05$ for differences between cell types by Kruskal-Wallis Test (Supplementary Table 6) in

825 GCs (A) and SGCs (B). Summary plot of cumulative charge transfer over one second (C) at

826 three developmental stages in both cell types. \#, and $\$$ denote $p<0.05$ for differences in GC

827 across age groups and SGC across age groups, respectively by TW-ANOVA followed by post-

828 hoc pairwise comparison.

829 Supplementary Figure 6: Developmental differences in distribution of sIPSC amplitudes in

830 GCs and SGCs. Pie chart distributions illustrating subjective percentage distribution of high 831 peak amplitude ( $>50 \mathrm{pA})$ and smaller peak amplitude ( $<50 \mathrm{pA})$ amplitude sIPSCs in GCs (top) 832 and SGCs (bottom) across age groups. 
833 Supplementary Figure 7: Analysis of developmental changes in passive membrane

834 properties of GCs and SGCs. Representative voltage traces in response to a -200pA current

835 injection for one second in GCs and SGCs at the developmental stages under investigation

836 reveal differences in $R_{\text {in }}(A)$. Summary of $R_{\text {in }}$ in the cell types (B). Summary plot of membrane

837 time constant ( $\tau_{\text {membrane }}$ ) obtained from single exponential fits to the voltage response to $-200 \mathrm{pA}$

838 current injection (C). *, \#, and $\$$ denote $\mathrm{p}<0.05$ for differences between cell types, in GC across

839 age groups and SGC across age groups, respectively by TW-ANOVA followed by post-hoc

840 pairwise comparison (Supplementary Table 8). 


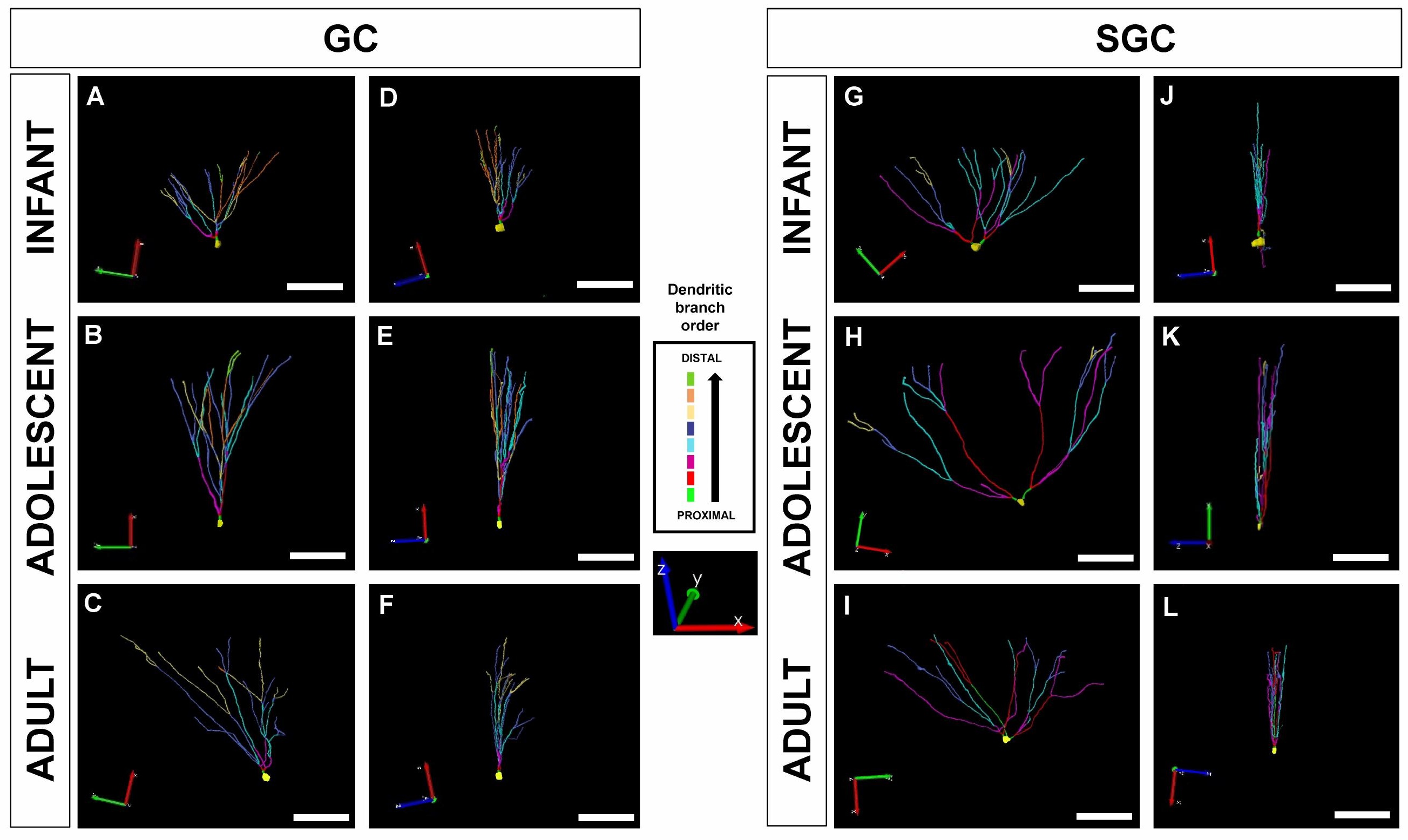




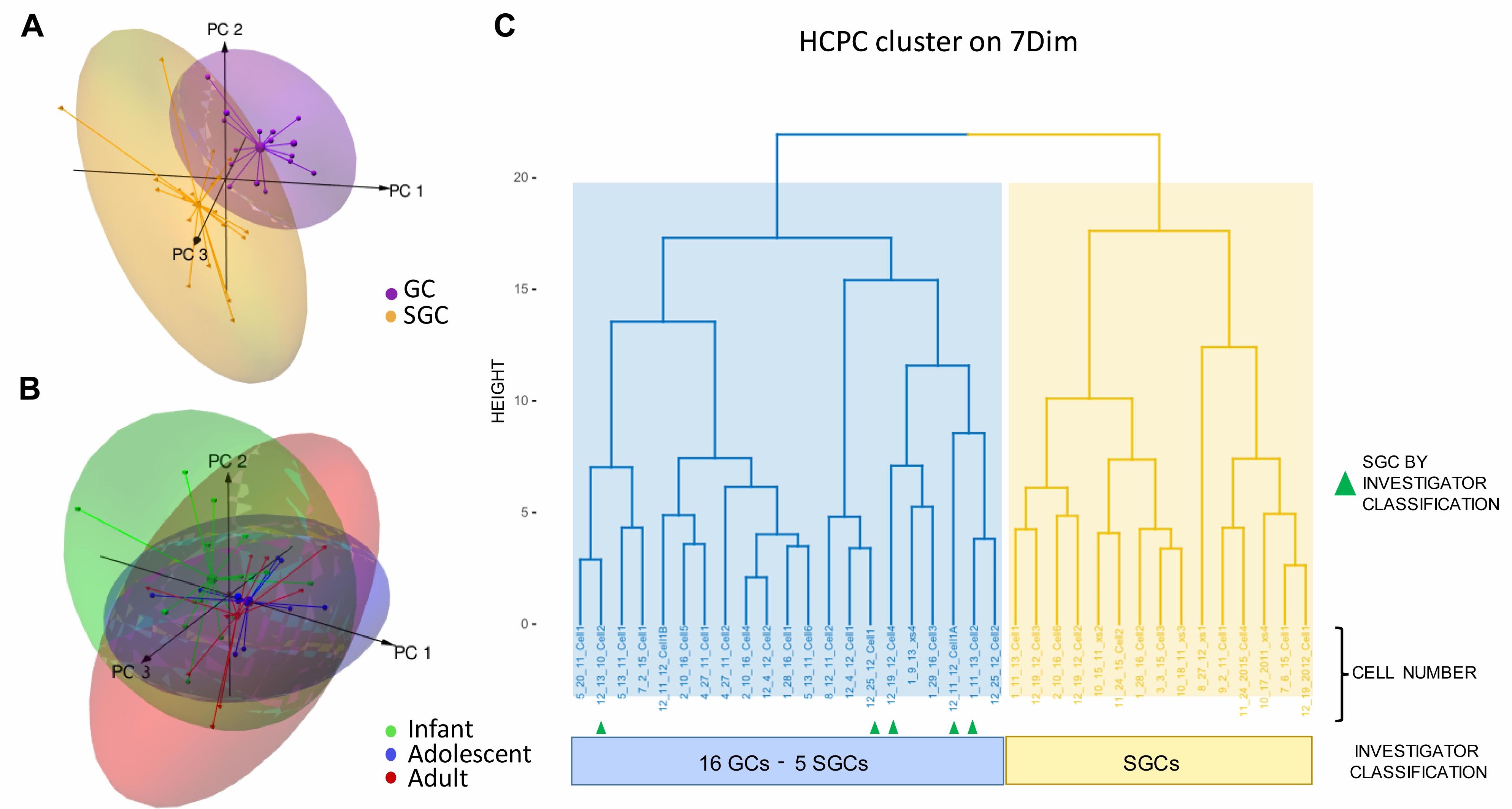




\section{Figure 3}

A
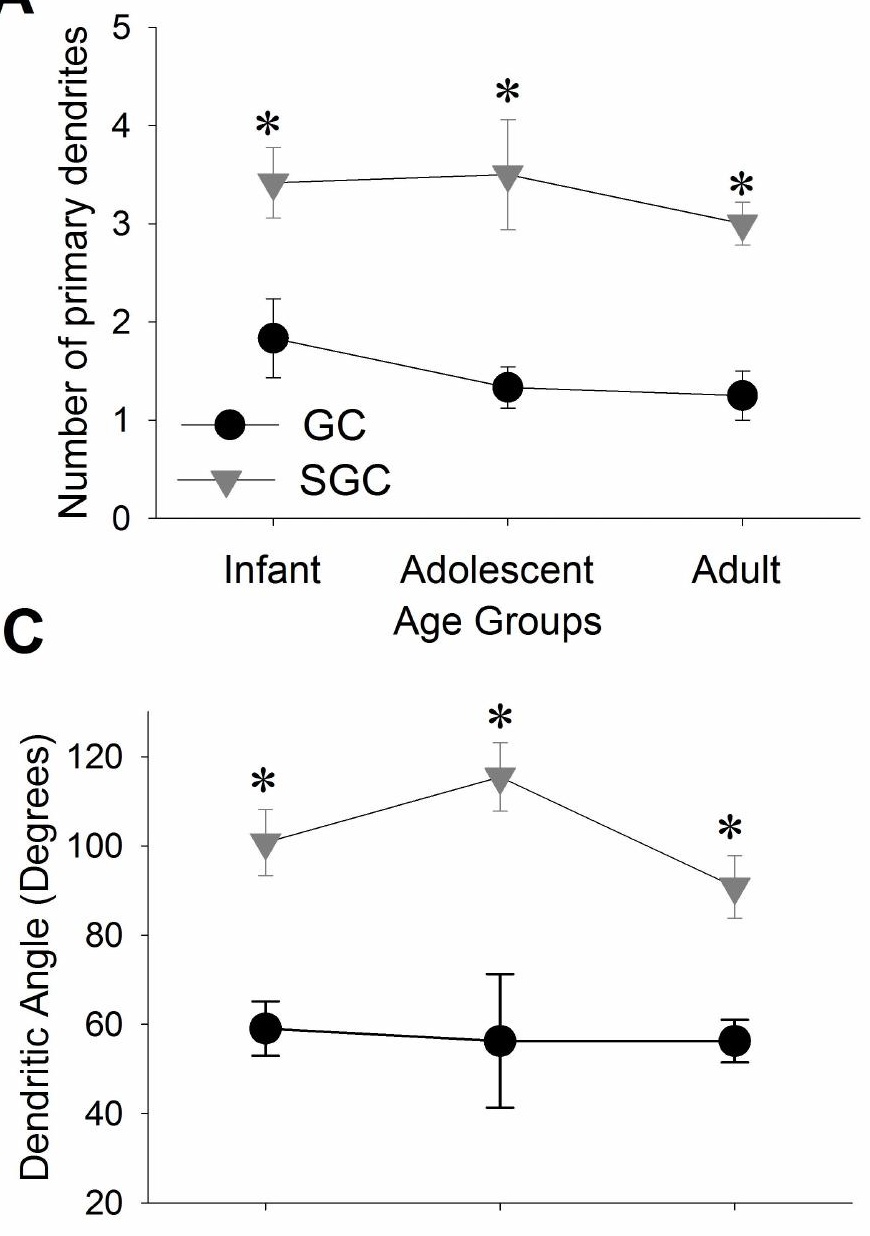

E

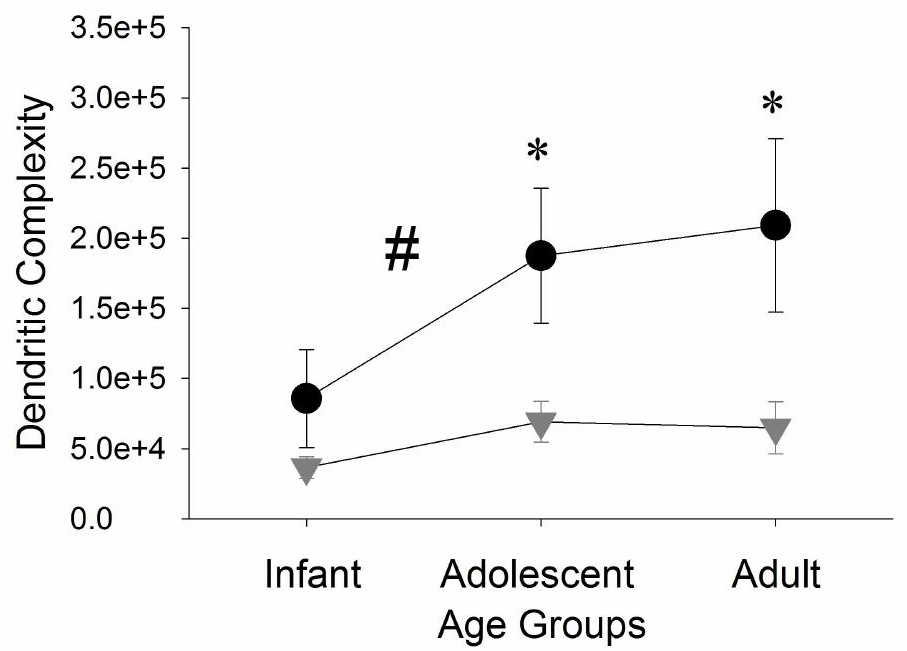

B
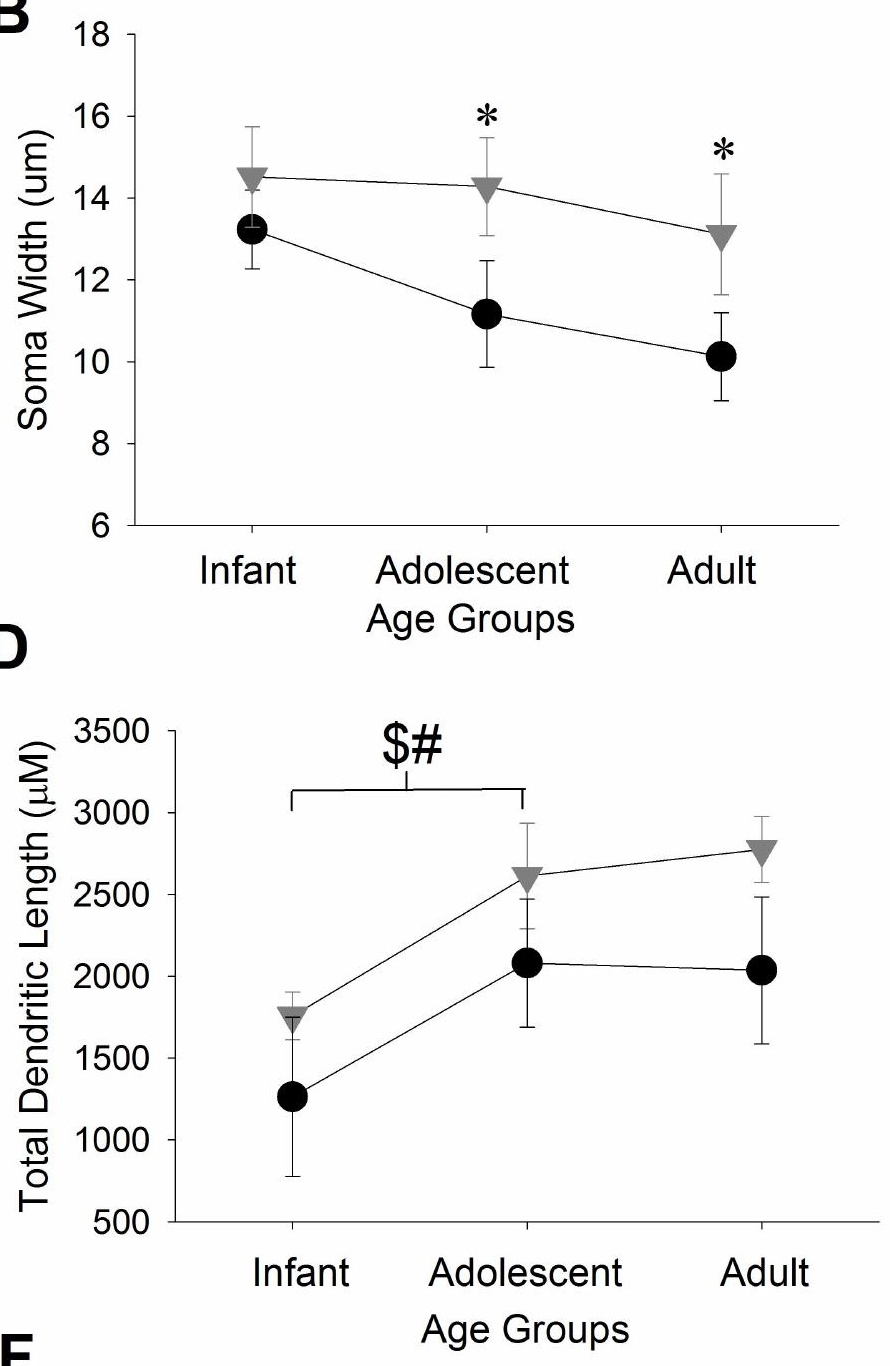

$F$

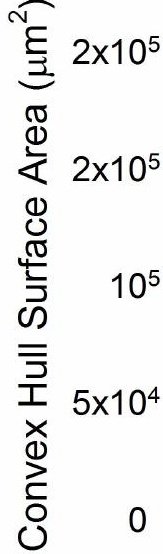


Figure 4

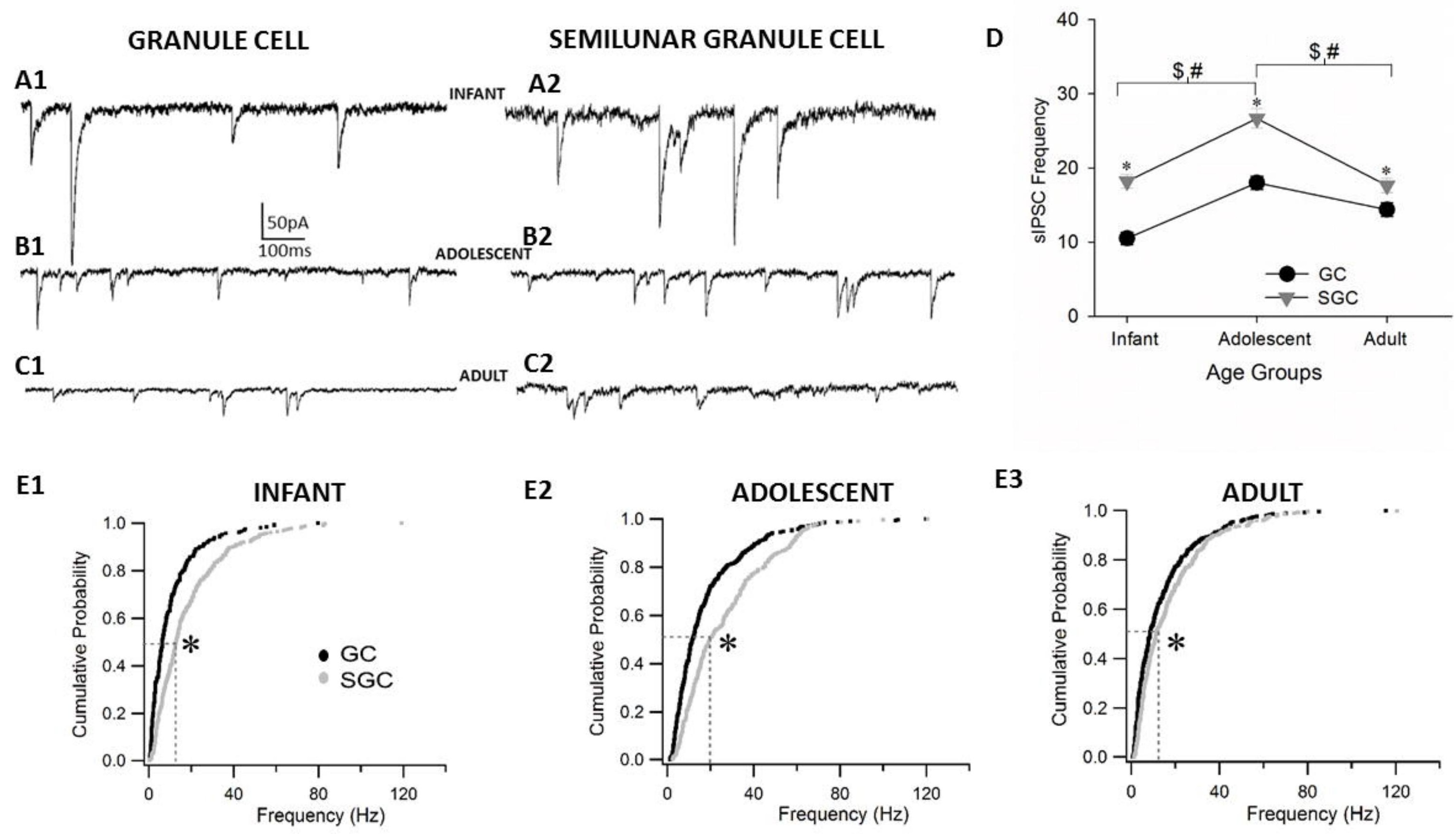


A

GC

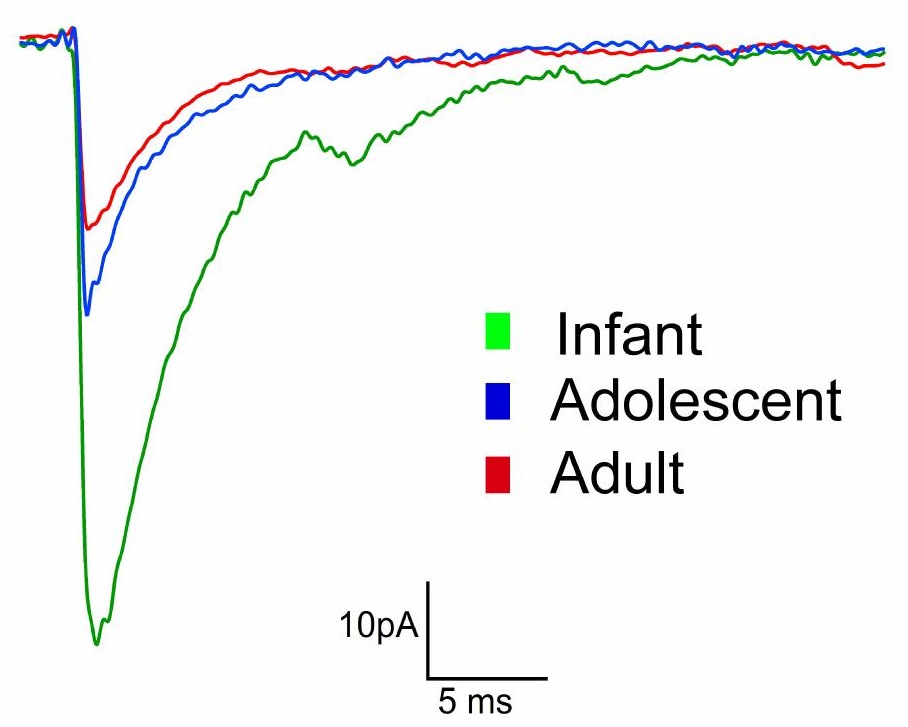

D

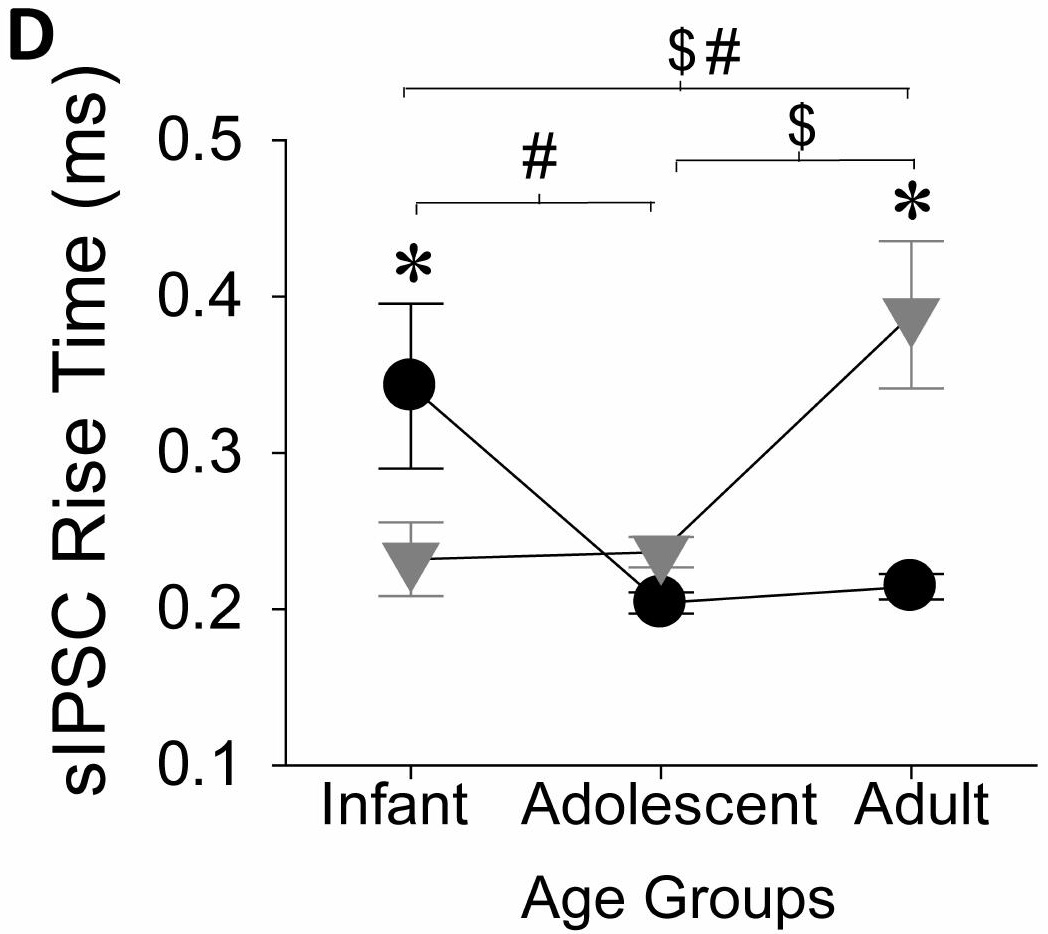

B

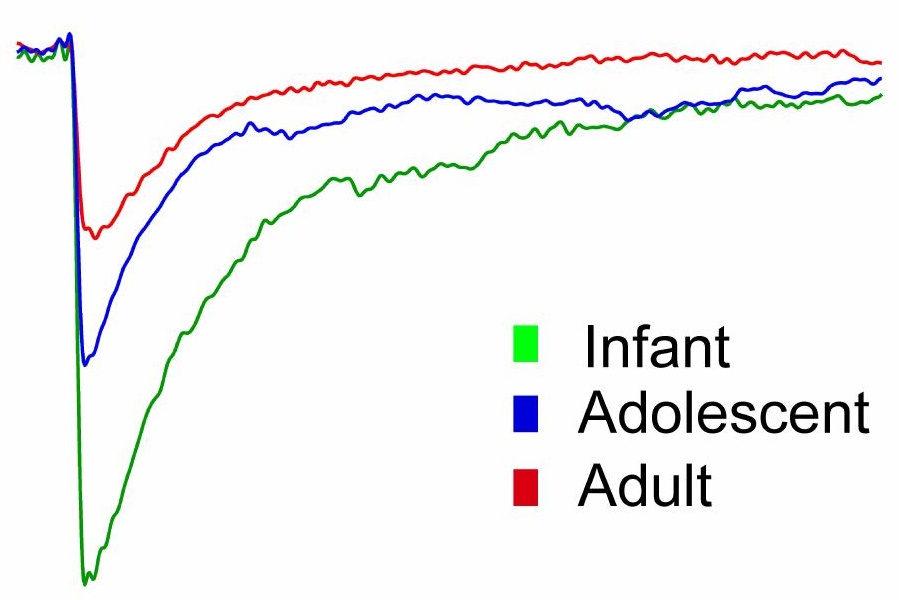

E

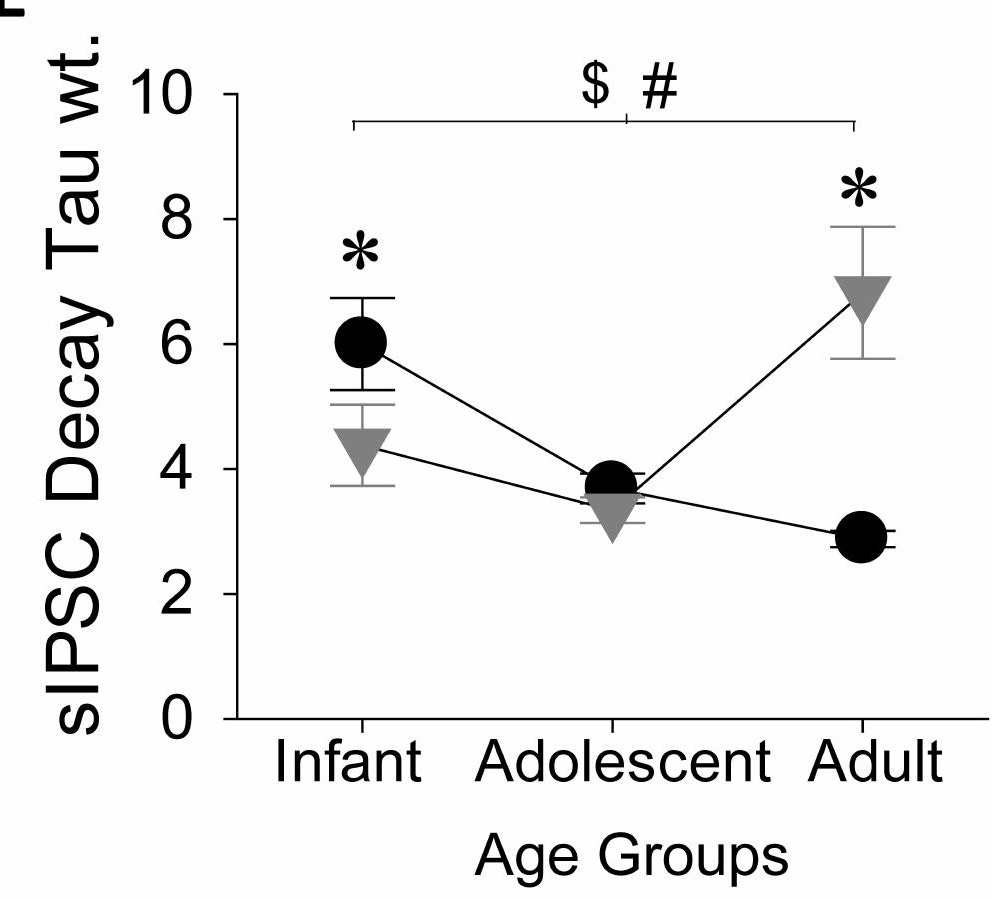

C

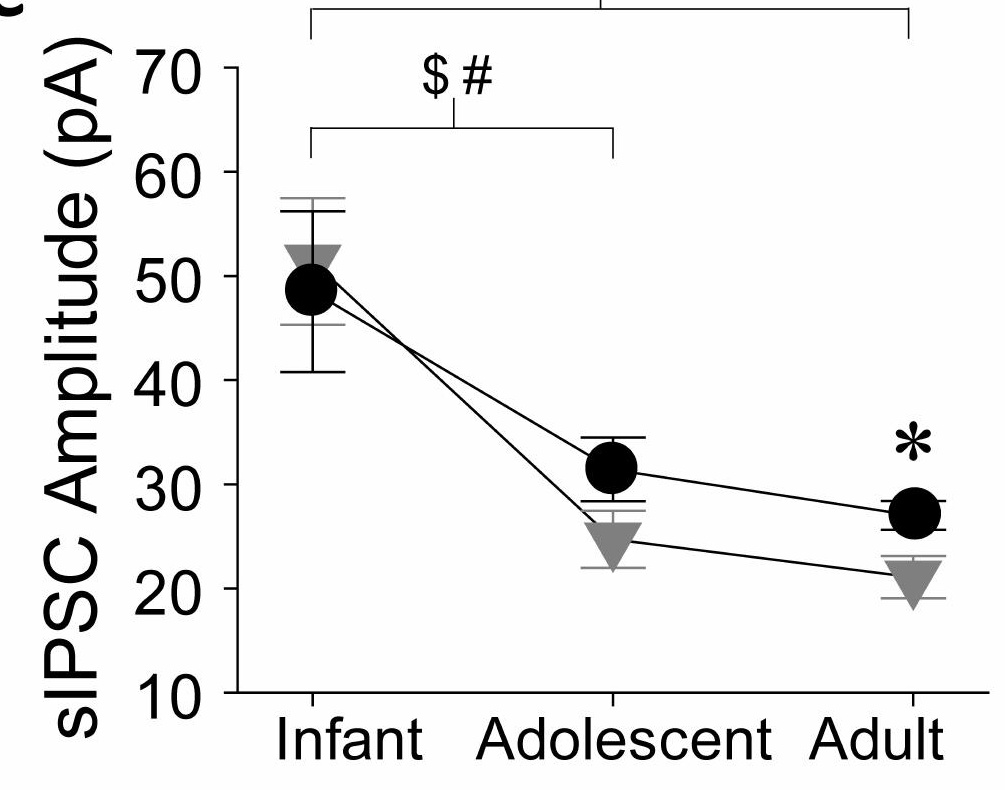

Age Groups

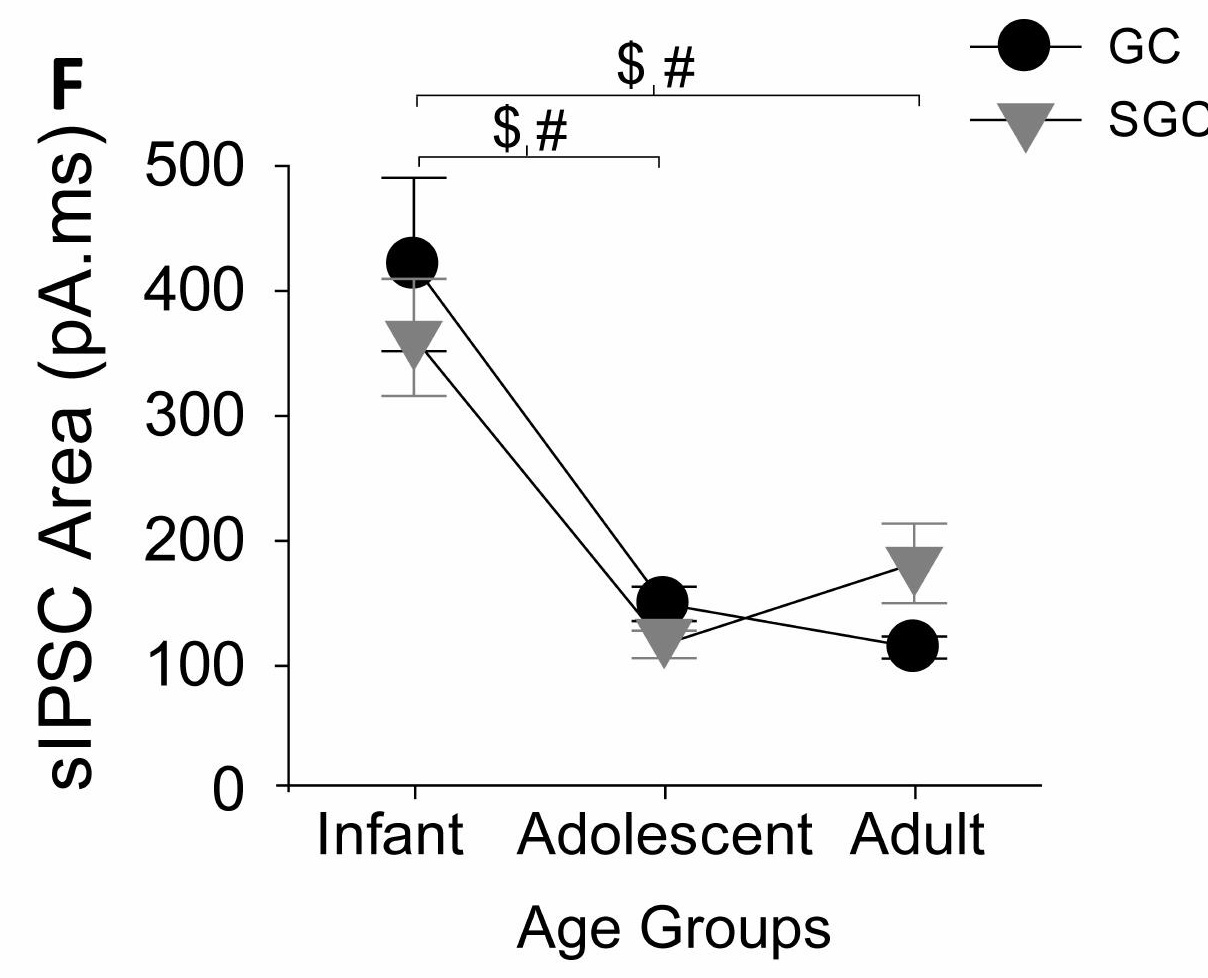




\section{GC}

\section{SGC}
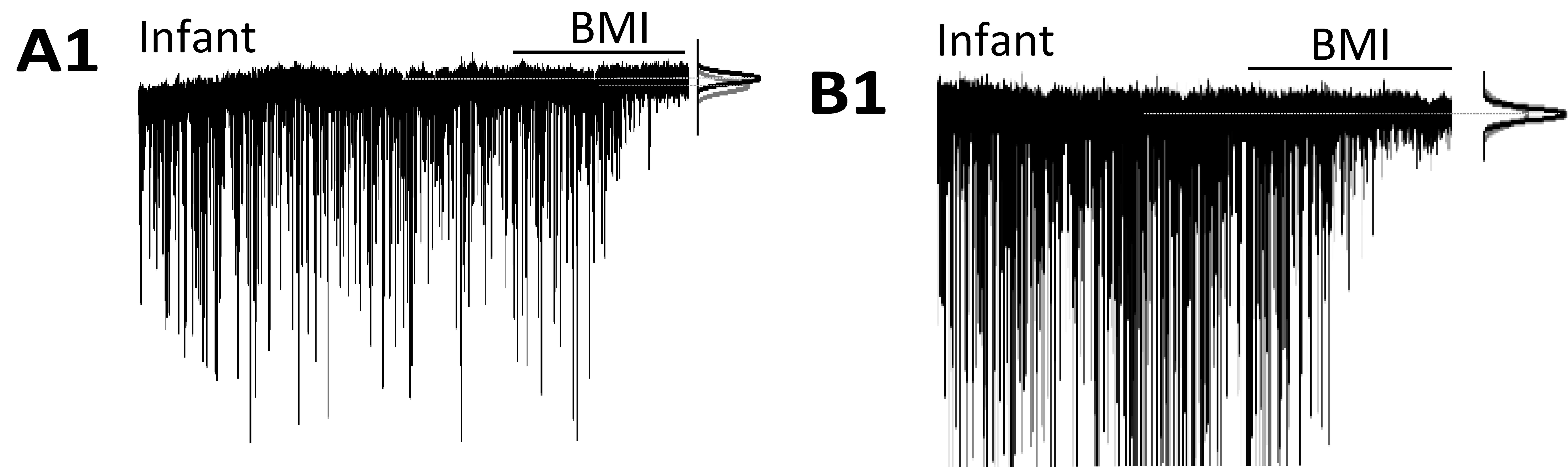

A2 Adolescent

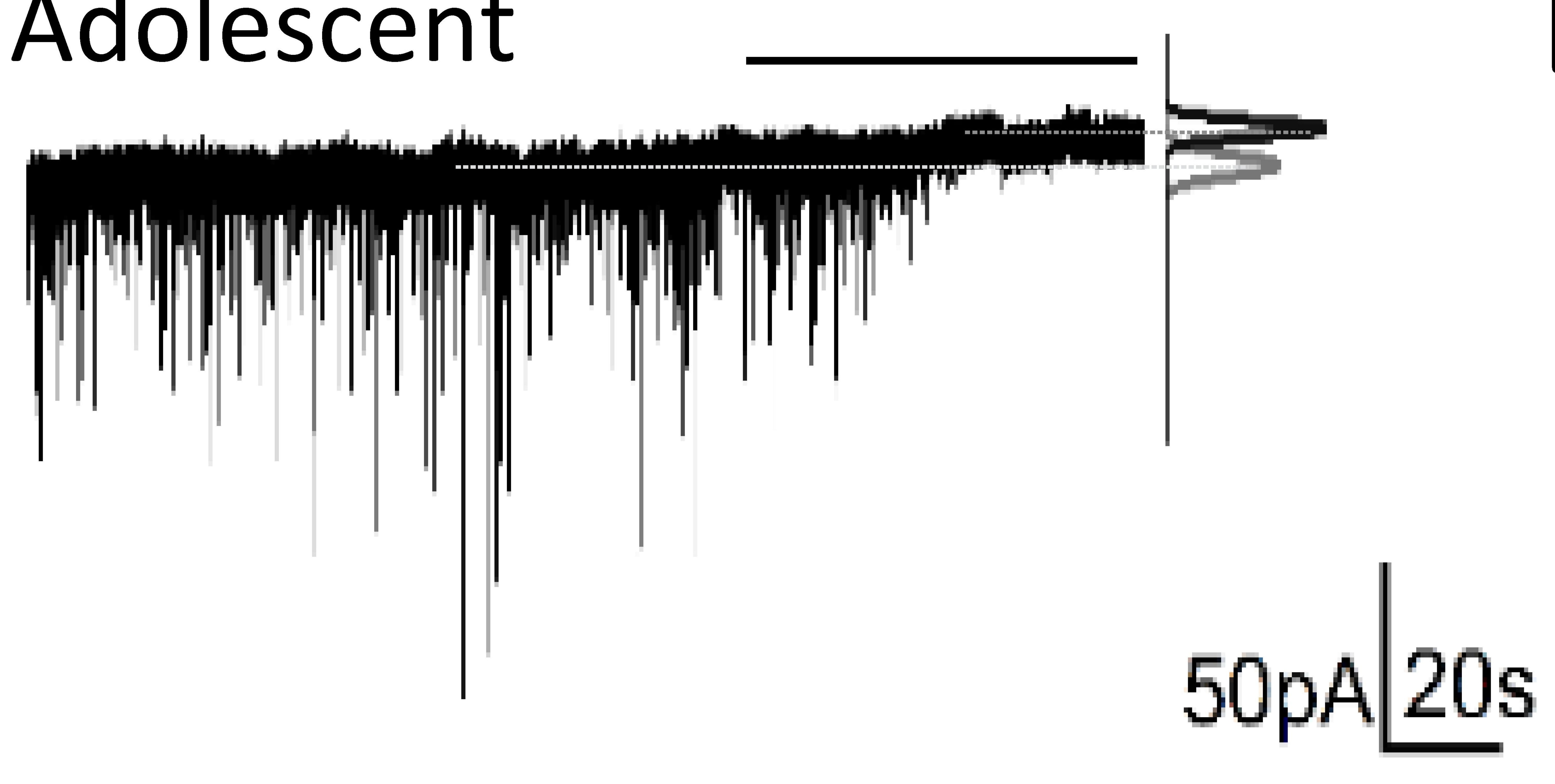

B2 Adolescent

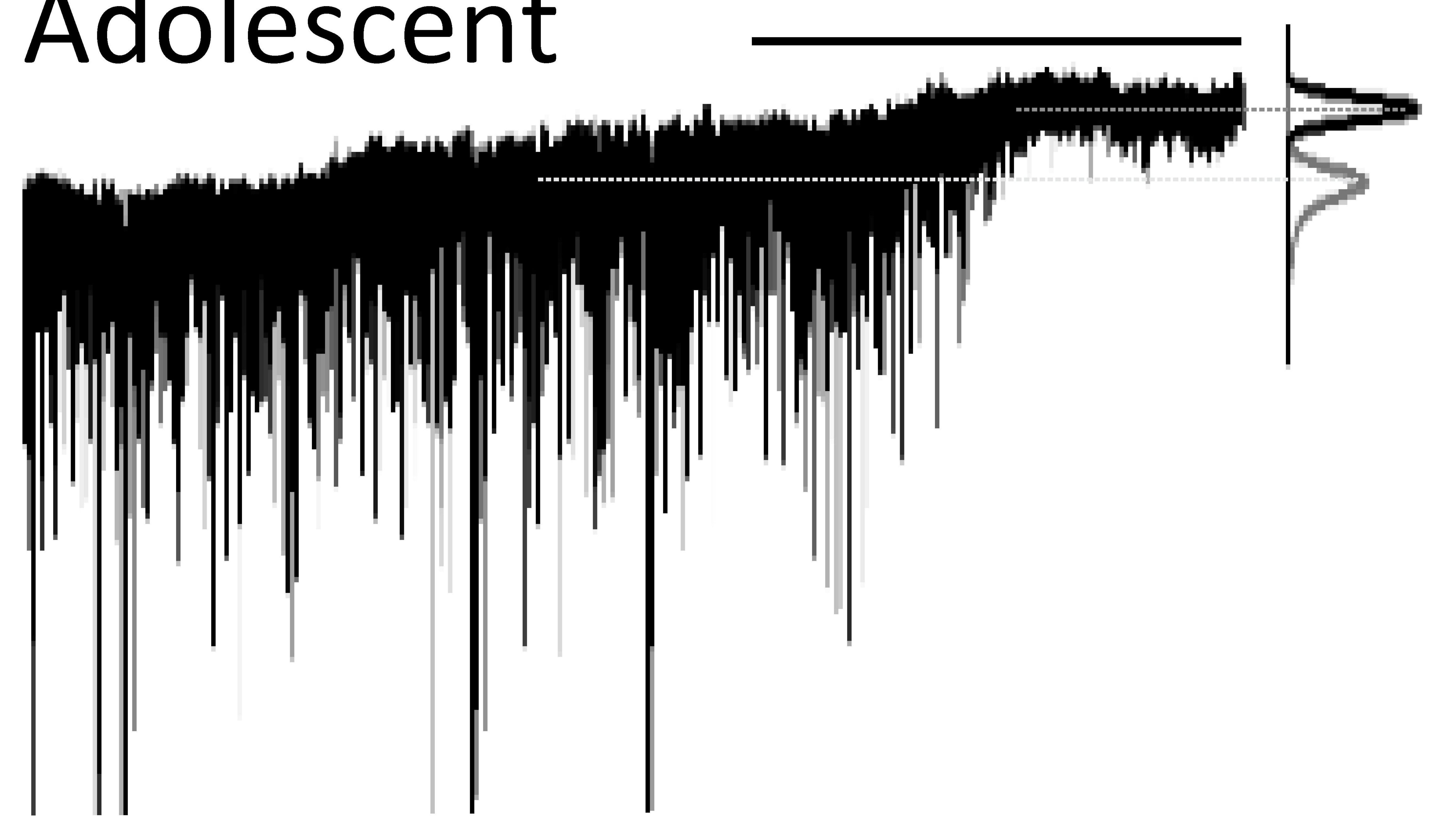

A3

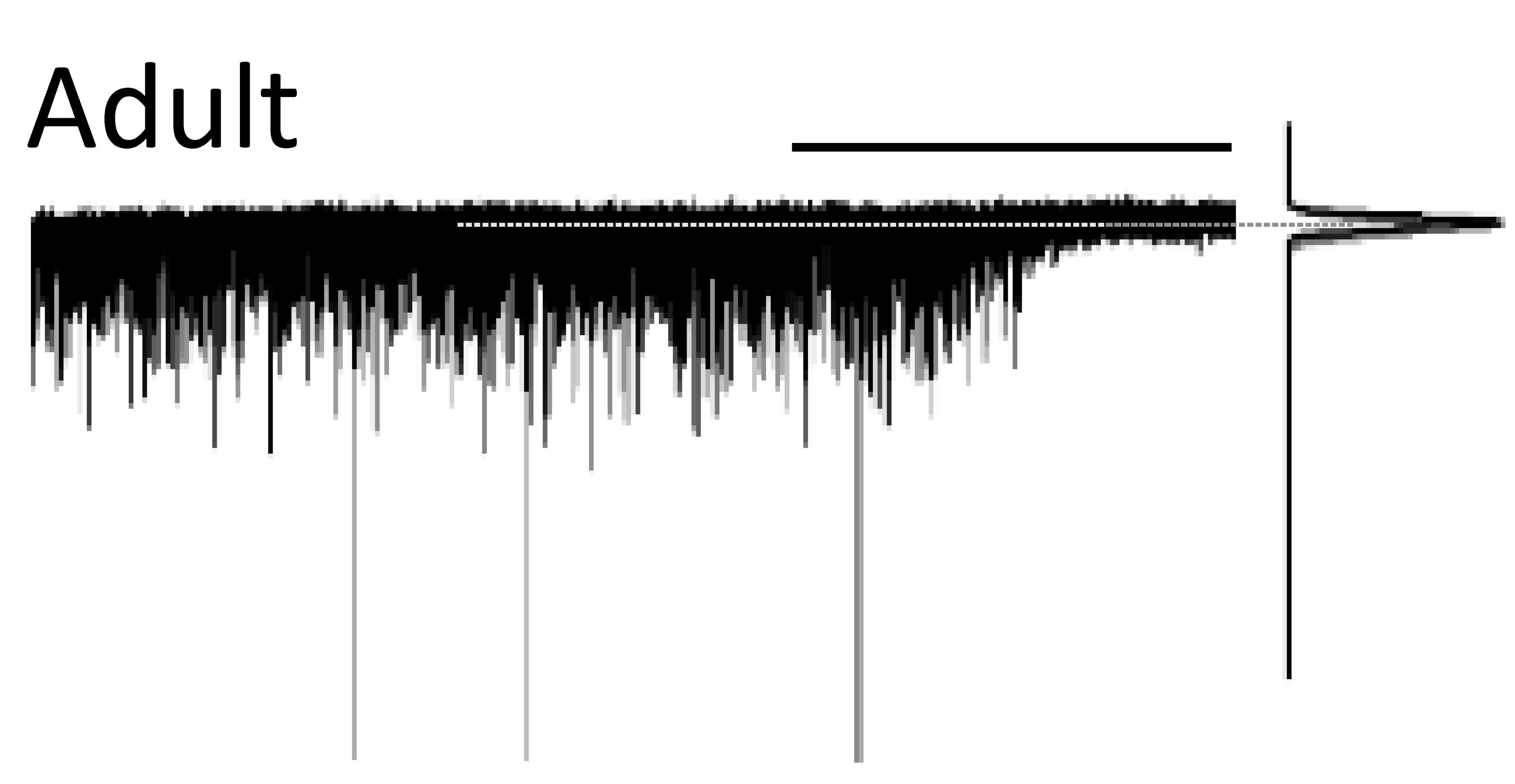

B3
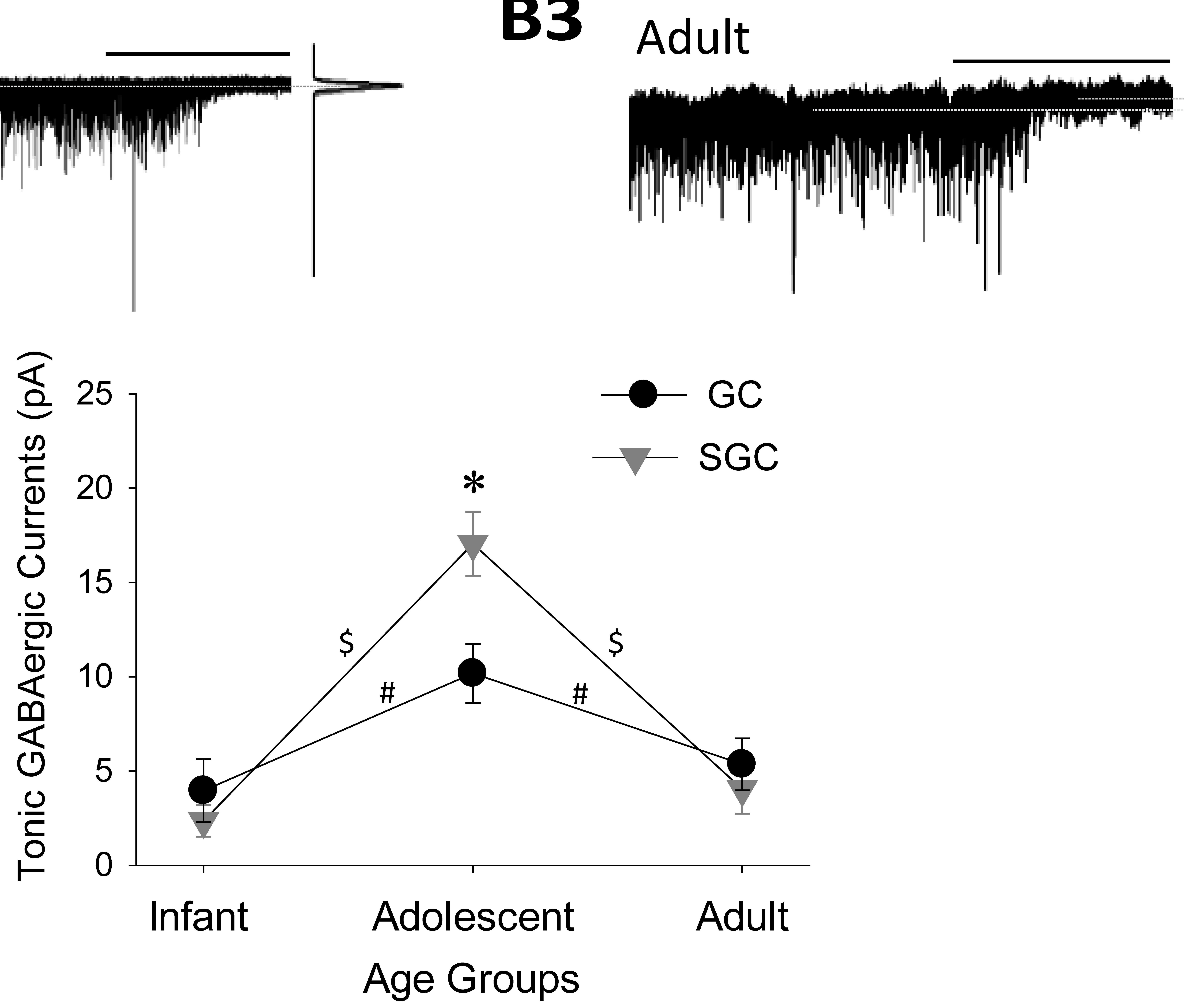